\title{
SCFOsFBK1 mediates JA induced turn-over of OsATL53 and OsCCR14 to regulate rice anther lignification
}

Pratikshya Borah ( $\square$ pratikshyaborah@south.du.ac.in )

Interdisciplinary Centre for Plant Genomics, University of Delhi South Campus https://orcid.org/00000003-2005-7963

Aishwarye Sharma

University of Delhi South Campus

Jitendra Khurana

University of Delhi

Article

Keywords:

Posted Date: January 26th, 2022

DOI: https://doi.org/10.21203/rs.3.rs-1267294/v1

License: (c) (1) This work is licensed under a Creative Commons Attribution 4.0 International License. Read Full License 
2 SCF ${ }^{\text {OsFBK1 }}$ mediates JA induced turn-over of OsATL53 and OsCCR14 to regulate rice anther

3 lignification

4

5 Authors: Pratikshya Borah ${ }^{1,2^{\wedge}+}$, Aishwarye Sharma ${ }^{\wedge}$ and Jitendra Paul Khurana ${ }^{1,2}$

$6 \quad 1$ Interdisciplinary Centre for Plant Genomics, University of Delhi South Campus, New Delhi $7 \quad 110021$, India

8 2Department of Plant Molecular Biology, University of Delhi South Campus, New Delhi - 110021, $9 \quad$ India

$\wedge$ Authors contributed equally

$11+$ To whom correspondence should be addressed: Pratikshya Borah, 12 pratikshyaborah@south.du.ac.in

13 ORCID ID: Pratikshya Borah: 0000-0003-2005-7963, Aishwarye Sharma: 0000-0003-3686-6319, 14 Jitendra P. Khurana: 0000-0003-3808-1662

15 Funding: The Department of Biotechnology, Government of India 16 (BT/PR12394/AGIII/103/891/2014) provided the financial support. J.P.K. also acknowledges the 17 award of J.C. Bose National Fellowship by the SERB, Government of India (SB/S2/JCB-13/2013);

18 P.B. availed the Research Associateship sanctioned as part of the DBT project. A.S. would like to 19 thank the University Grants Commission, New Delhi, for the award of a Research Fellowship. For 20 infrastructural support, authors acknowledge the financial assistance provided by the Department 21 of Science and Technology (through FIST and PURSE Grants), the University Grants Commission 22 (through UGC-SAP grant) and the University of Delhi. This research work is dedicated to late JPK, 23 a great mentor, brilliant scientist, and a gentle human being. 
ABSTRACT

25 We have shown earlier the F-box protein, OsFBK1, mediating turn-over of a cinnamoyl CoA26 reductase, OsCCR14, to regulate rice anther lignification. Currently, we have identified OsATL53, 27 a member of ATL family of RING-H2 proteins interacting with OsCCR14 in cytoplasm. OsFBK1 mediates turn-over of OsATL53 in cytoplasm and nucleus, while of OsCCR14 in the nucleus. In presence of jasmonic acid (JA), which plays a role in anther dehiscence, FLIM analyses demonstrate OsATL53-OsCCR14 undergoing conformational changes that trigger the complex to accumulate around the nuclear periphery and signals OsFBK1 to initiate degradation of the proteins in respective cellular compartments. Biochemically, OsATL53 decreases enzymatic activity of OsCCR14 and sequesters it in the cytoplasm. Knock-down transgenics of OsATL53 display increased lignin deposition in the anthers vis-a-vis wild-type, while those of OsCCR14 have decreased lignin content. These data show OsATL53 affects activity of OsCCR14, and their JA induced degradation by SCFOsFBK1 regulates lignification of rice anthers.

\section{INTRODUCTION}

Homeostasis in protein levels in all biological processes is a critical and essential regulatory mechanism. The onus of this regulation falls vastly on the ubiquitin $26 \mathrm{~S}$ proteasome-mediated system (UPS) that is conserved across species. The ligases in the UPS participate in a range of concerted processes including substrate identification to mediating controlled ubiquitination of target proteins. The specificity in target protein recognition is particularly expanded in plants where there is a presence of an enormous number of E3 ligases as compared to animals (1), (2). Plant E3 ligase genes vastly outnumber those of the other non-plant eukaryotes, where approximately 1400 E3 ligase encoding genes have been annotated in Arabidopsis thaliana genome and about

47600 in the human genome (3), (4). Of the different classes of E3 ligases available, the RING-type 48 family forms the largest group. The RING family of E3 ligases is characterized by the presence of 
49 the canonical RING domain that functions as an intermediary between E2-ubiquitin (Ub) and the

50 E3 ligases. RING-based E3 ligases could be either single-subunit or multi-subunit. The cullin-RING

51 E3 ligases (CRLs) are complex RING-type E3 ligases and amongst them the most abundant type

52 found in plants is the SCF type (3), (5).

53 The RING-finger domain found in RING-type E3 ligases is a specific type of zinc finger that binds

54 two Zn ions in a 'cross-brace' structure using a defined pattern of cysteine $(\mathrm{C})$ and histidine $(\mathrm{H})$

55 residues found in its conserved aā sequence (6). The $\mathrm{C}_{3} \mathrm{HC}_{4}(\mathrm{RING}-\mathrm{HC})$ and $\mathrm{C}_{3} \mathrm{H}_{2} \mathrm{C}_{3}$ (RING-H2)

56 are the most common RING domains where RING-HC domain has been found in single

57 polypeptide E3 ligases and RING-H2 domain is associated with complex multi-subunit E3 ligases

58 (7). Apart from these domains, the presence of less frequent domains, like RING-v, RING-C2,

59 RING-D, RING-S/T and RING-G type, has also been described (8). A staggering 551 potential E3

60 ligases have been reported in Arabidopsis thaliana where 451 correspond to RING-H2 variant and

61 the proportion of this variant in species like Brassica rapa and Oryza sativa are 55\% and 57\%,

62 respectively $(9,10)$. It has been previously reported that some RING zinc finger protein genes are

63 rapidly induced in response to elicitors and these genes are members of a family known as ATL

64 (Arabidopsis Tóxicos en Levadura) (11). Mutants of ATLs have been previously isolated and

65 analysed to reveal a link between these putative ubiquitin (Ub) ligases and plant defence signalling

66 pathways (12). It was also reported that the Arabidopsis family consisted of 80 members and rice

67 had 121 ATL genes (13). In the past years, two new classes of RING E3 ligases have been

68 identified in addition to ATLs, based on RING-H2 domain variations and RING-outside conserved

69 motifs: BCA 2 Zinc-Finger (BZF) ATLs (BTLs) (14) and CTLs containing the YEELL conserved

70 motif (15).

71 Several E3 RING-finger protein genes have been characterized over the years for their role in

72 diverse processes; these include CONSTITUTIVE PHOTOMORPHOGENIC 1 (COP1) in light

73 signalling $(16,17)$, SINA of Arabidopsis thaliana 5 (SINAT5) in accentuating auxin signalling (18, 
19), HIGH EXPRESSION OF OSMOTICALLY RESPONSIVE GENES 1 (HOS1) in cold response

75 (20), ACCUMULATION AND REPLICATION OF CHLOROPLASTS 1 in self-incompatibility (21),

SHOOT APICAL MERISTEM ARREST 1 involved in shoot apical meristem maintenance (22), ABI1-INTERACTING PROTEIN 2 in ABA signalling (23), RING-H2 GROUP F1A/RING-H2 GROUP

F2A in gametogenesis (24) and DEFECTIVE IN ANTHER DEHISCENCE 1 (DAD1)- ACTIVATING

FACTOR (DAF) that affects jasmonic acid (JA) biosynthesis (25), to name a few. Several ATL genes that have been studied include ARABIDOPSIS TÓXICOS EN LEVADURA 2 (ATL2), which participates in plant defence (12), ELICITOR 5 (EL5) that regulates cell death during root development, and DAY NEUTRAL FLOWERING (DFN)/AthATL62, in repressing photoperiod response (26). Some of the important CTLs characterized include Arabidopsis E3 ligase BIG BROTHER $(B B)$ regulating organ size (27); the human RNF11/ARKADIA that is a TGF-signalling and DNA damage responsive SUMO E3 ligase gene (28); RNF 165/ARK2C (29) and the p53 regulator $R N F 38(30)$.

\section{RESULTS} ligase and interacts with OsCCR14 (OsCCR20 (31)), the substrate of the F-box protein, OsFBK1. These two proteins were identified as putative substrates of OsFBK1 in a $\mathrm{Y} 2 \mathrm{H}$ anther library screening, and degradation of OsCCR14 by SCFOsFBK1 has been described to regulate lignification in rice anthers and roots (32). We have tried to explore the degradation mechanism of protein factors (involved in lignification) in response to hormones like jasmonate, as well as provide a new insight into the function of RING-H2 ATL proteins.

\section{OsATL53 is a component of E3 ligase and interacts with both OsFBK1 and OsCCR14}

OsATL53 (RING-H2 protein) is one of the 121 odd RING-H2 ATLs found in rice with a distinct signature sequence (13). Sequence alignment between OsATL53 and known RBX1 proteins studied in humans, yeast and Arabidopsis has also shown that there is homology amongst these 
99 proteins (Fig. S1A), even though OsATL53 is not the direct homologue of the canonical RBX1 in reverse blast hits. ATL proteins are also known to comprise of an $\mathrm{N}$-terminal region that is rich in hydrophobic amino acids, and the module generally comprises of a single hydrophobic region of at least 18 residues (13). OsATL53 also has been found to contain one $\mathrm{N}$-terminal hydrophobic transmembrane domain of 22 amino acid residues (Fig. S1B).

104 105 106

To confirm whether OsATL53 could be a component of SCF E3 ligase, in vitro immunoprecipitation assay was carried out between GST-CUL5g (32) and 6xHis-OsATL53, and a weak interaction was detected (Fig. 1A). We had previously shown that the SCFOsFBK1 E3 ligase complex comprises of OSK1/20, CUL5g (non-standard nomenclature) and OsFBK1 employing Y2H assay (32). With the current data it appears that the complete SCFOsFBK1 also harbours OsATL53, probably as the RBX1 component (Fig. S1D), although it cannot be ruled out that OsATL53 can be a part of another RING-based E3 ligase.

A detailed study on the architecture of ATLs has suggested that there are two distinct regions in ATLs that are responsible for mediating protein-protein interactions; region I located at the aminoterminal end that interacts with components of E3 ligase, and region VII positioned towards the carboxy-terminal end that might be involved in target recognition (33). To test this hypothesis with OsATL53, we generated GST-tagged deletion constructs having a truncated N-terminus, $\triangle$ OsATL53 (region I, including the transmembrane domain, amino acids from 1 to 32 ) and a truncated C-terminal end, OsATL53 $\Delta$ (region VII, amino acids from 138 to 202), respectively (Fig. S1B).

ATLs like DAF (DEFECTIVE IN ANTHER DEHISCENCE1-Activating Factor) have been previously described to have auto-ubiquitination activity (25). First, we investigated the auto-ubiquitination activity of OsATL53 using a modified protocol described earlier (25). As is evident from Fig. 1B, OsATL53 has auto-ubiquitination activity and can probably function as an E3 ligase, while the truncated constructs functioning as negative controls showed little or no ubiquitination activity. 
$124 \triangle$ OsATL53 failed to display any auto-ubiquitination property, while OsATL53 $\Delta$ showed diminished 125 activity. These results also show that region I of OsATL53 is involved in ubiquitination activity. We 126 also observed a slight shift in size without an apparent smear in the ubiquitinated form of OsATL53 127 (lane 4 of Fig. 1B) indicating that the addition of ubiquitin is probably in the mono form rather than 128 poly-ubiquitination.

129 In general, RING-H2 proteins have been found to localise to different cellular compartments (34, 35) and OsATL53 has been found to specifically localize to the cytoplasm of onion epidermal cells 131 and absent in the nucleus (Fig. 1C and Fig. S3). For all localisation experiments using confocal 132 microscopy, we chose to work with onion epidermal cells as it is a monocot system, has a visibly 133 distinguishable nucleus and is a standardized and widely used transient expression system. As 134 stated earlier, OsATL53 was identified as one of the putative substrates of OsFBK1 in the $\mathrm{Y} 2 \mathrm{H}$ 135 anther library screening along with OsCCR14 (32). This interaction was further confirmed by in 136 vitro immunoprecipitation assay between GST-OsFBK1 and 6xHis-OsATL53 (Fig. 1D) and by split 137 EYFP BiFC (Fig. 1E). However, the BiFC interaction was found to be labile and required short 138 incubation time after bombardment (10-12 h). While OsATL53 is localised solely to the cytoplasm, 139 BiFC between OsFBK1 and OsATL53 showed a dominant cytoplasmic interaction along with a 140 slight presence of the complex in the nucleus (nuclear plane image, Fig. 1E); implying that the 141 interaction between these proteins takes place in both cellular compartments, albeit with a 142 cytoplasmic preference.

143 In a serendipitous experiment, OsATL53 was found to interact with OsCCR14 with BiFC EYFP 144 fluorescence found scattered throughout the cytoplasm of the onion peel cells (Fig. 1E). BiFC was 145 found to be stable even after $16 \mathrm{~h}$ post incubation after particle bombardment. This scattered 146 appearance of fluorescence, however, did not match with any organellar localisation (data not 147 shown) and resembles protein aggregates. This interaction was further confirmed by in vitro 148 immunoprecipitation (Fig. 1F). OsATL53, however, does not interact with OsCCR18 (OsCCR19 
149 (31), Fig. S1E), the closest homologue of OsCCR14 (32), indicating that the OsATL53-OsCCR14 150 interaction is specific.

151 Next, in vitro immunoprecipitation assays were carried out with OsFBK1 and OsCCR14 separately 152 using both OsATL53 deletion constructs. Western blot analyses show $\triangle$ OsATL53 interacting 153 weakly with OsFBK1, but its interaction with OsCCR14 is unaffected, indicating that region I is 154 involved in associating with the components of the functional ubiquitin ligase. Whereas for 155 OsATL53 5 , the interaction with OsCCR14 (the target of SCFOsFBK1) is vastly hampered, indicating 156 that the C-terminal domain of OsATL53 is involved in target recognition as reported earlier (33). 157 These experiments also strengthen the idea that RING-H2 proteins might have multiple roles apart 158 from their property to function in the ubiquitination process per se. Putative models of OsATL53159 OsCCR14 and OsFBK1-OsATL53-OsCCR14 interactions were generated by ab initio modelling 160 (Fig. S2), and the models were checked by Ramachandran plot analyses.

161 OsATL53 is a substrate of OsFBK1 and is preferentially degraded in the cytoplasm

162 Cell-free degradation assays using the total plant protein extracts of 7 -day-old seedlings of wild163 type (WT), vector control (VC) and OsFBK1 transgenics as described earlier (32) demonstrate that 164 OsATL53 degradation is mediated by OsFBK1 via the 26S proteasome pathway (Fig. 2A and 2B). 165 BiFC fluorescence showed OsFBK1-OsCCR14 interaction preferentially visible in the nuclei, with 166 weak signals detectable in the cytoplasm (Fig. 2C; see also (32)), while that of OsFBK1-OsATL53 167 was detected predominantly in the cytoplasm with weak signals visible in the nucleus (Fig. 1E and 168 2E). Such interactions in specific cellular compartments could provide hint at the target proteins' 169 sites of degradation mediated by SCFOsFBk1 E3 ligase. As proof, we carried out separate cell-free 170 degradation assays of 6xHis-OsCCR14 and 6xHis-OsATL53 in both nuclear and cytoplasmic 171 protein extracts of OsFBK1OE and WT lines. Fig. 2C and 2D show degradation of OsCCR14 by 172 SCFOsFBK1 taking place specifically in presence of nuclear protein-enriched extracts under the given 173 experimental duration; while Fig. $2 \mathrm{E}$ and $2 \mathrm{~F}$ show that OsATL53 is degraded in both protein 
174 fractions but preferentially in the cytoplasmic protein fraction. Hence, the BiFC signals observed for

175 both OsCCR14 and OsATL53 with OsFBK1 indeed indicate the sites of interaction where their

176 subsequent degradation takes place. The labile split-EYFP signals observed for OsFBK1-

177 OsATL53 interaction (Fig. 1E) also correspond to the faster degradation kinetics of OsATL53

178 mediated by SCFOsFBK1. That cytosolic enzymes like OsCCR14 are degraded specifically in the

179 nucleus and mediated by F-box proteins find support from some earlier studies in animal systems 180 (36).

\section{Jasmonic acid changes the dynamics of the OsCCR14-OsATL53 complex}

182 The process of anther dehiscence preceding pollination is characterised by three distinct events

183 (37). During anthesis, auxin regulates commencement of anther dehiscence by controlling the 184 timing of the lignification at the start of late stamen development (38), while JA is thought to initiate 185 dehydration and opening of anther at the final stages of anther development (39-43). Thus, it has 186 been suggested that an auxin maximum at early floral stages in Arabidopsis blocks premature endothecium lignification, and its decline in the later stages triggers endothecium lignification. This reduction in the auxin concentration also leads to the increase in JA concentration, thereby causing stomium breakage and anther dehiscence (44).

Though the degradation of OsCCR14 (the enzyme catalysing the second rate-limiting step in lignin formation) by SCFOsFBK1 is not affected by auxin, its transcription is induced by auxin (32).

192 However, as JA affects the process of anthesis, it might regulate the degradation of OsCCR14 to 193 affect anther lignification. Thus, we first carried out particle bombardment on onion peel cells using 194 various constructs (Fig. S3) and treated the peels with $6.4 \mathrm{mM} \mathrm{JA}$ for $4 \mathrm{~h}$ to check whether JA 195 affects the localization of these proteins (Fig. S3). The concentration of JA used in our assay was 196 as deployed earlier (44) that triggered precocious anthesis in Arabidopsis faster than that observed 197 before (42). Since $6.4 \mathrm{mM} \mathrm{JA}$ is considerably higher than normal physiological conditions, we first 198 tested the effect of this concentration of JA on various constructs. We found that the localization of 
OsFBK1, OsCCR14 and the vector controls were unaffected by JA, even after $4 \mathrm{~h}$ of incubation. F-

200 boxes are known to function as hormone receptors (3), but since localization of OsFBK1 was unaffected after JA treatment (Fig. S3), we can presume that OsFBK1 does not function as a JA sensor. However, the EYFP fluorescence of OsATL53 appeared to accumulate around the nuclear periphery (Fig. S3 and Fig. S4; for cells with nuclear stain). This accumulation of OsATL53 around the nucleus was seen to initiate as early as $2 \mathrm{~h}$ post incubation (data not shown). In order to rule out the effect of stress caused by a high JA concentration in the bombarded cells during the experimental time frame, we checked the changes in lifetime of EYFP-OsATL53 on exposure to a 6.4 mM JA by FLIM (Fluorescence Lifetime Imaging) confocal microscopy (Fig. S5B), as the lifetimes of fluorophores are also affected by the cellular $\mathrm{pH}$ and ionic changes (45). Even after $4 \mathrm{~h}$ of incubation with $6.4 \mathrm{mM} \mathrm{JA}$, the lifetime of EYFP-OsATL53 did not change (Fig. S5C). A representative stressed or plasmolysed cell expressing EYFP-OsATL53 displays a decrease in lifetime due to changes in cell integrity (Fig. S5B and S5C). Further, we also checked the changes

212 in localization of EYFP-OsATL53 with $100 \mu \mathrm{M} \mathrm{JA}$, as done earlier (42) and found changes in its 213 localization initiating only after $8 \mathrm{~h}$ of incubation (Fig. S4A). Due to the shorter incubation time and 214 that the concentration does not affect the cellular integrity, we chose to use $6.4 \mathrm{mM} \mathrm{JA}$ for all 215 further experiments.

216 Interaction between OsATL53 and OsCCR14 was also analysed by co-localization using respective constructs and subjecting the bombarded cells to JA treatment for $4 \mathrm{~h}$. Fig. 3A shows a distinct accumulation of both EYFP (OsATL53) and ECFP (OsCCR14) fluorescence around the nucleus after $4 \mathrm{~h}$ of JA treatment. The localization of OsCCR14 is otherwise unaffected by JA (Fig. S3). This implies that JA affects localization of the OsCCR14-OsATL53 complex, presumably mediated by the movement of OsATL53 towards the nucleus on exposure to JA (Fig. 3A). On the

222 other hand, co-localization of OsFBK1-OsATL53 and OsFBK1-OsCCR14 were unaffected even in 223 the presence of JA (Fig. S6). However, OsATL53 was found to be present slightly in the nucleus when co-localized with OsFBK1 with or without JA (Fig. S6). This observation for OsCCR14- 
225 OsATL53 complex localization in response to/without JA was also repeated by BiFC (Fig. 3B).

226 However, no BiFC fluorescence beyond $4 \mathrm{~h}$ of JA exposure was observed.

227 We also investigated whether JA also induces structural changes in the OsATL53-OsCCR14 228 complex. For this we adopted the FLIM-FRET technique to examine any changes in the lifetime of 229 the ECFP-donor construct with/without JA. We used FRET probes that have both the fluorescent 230 probes (ECFP and EYFP) in the N-terminal regions as opposed to the combination used in BiFC, 231 and technically will be separated beyond the required FÖrster distance $(<10 \mathrm{~nm})$. As expected, the 232 lifetime of the donor ECFP ( 3 ns in solution, https://www.fpbase.org/,(46)) did not change under 233 FLIM-FRET condition (Fig. 3C and 3D). However, when we added JA and incubated the peel cells 234 for 2 hours, we noticed that the lifetime of the donor ECFP had decreased indicating a positive 235 FRET (Fig. 3C and 3D). Positive FRET was not only observed around the nucleus where the 236 ECFP-OsCCR14 and EYFP-OsATL53 fluorescence were found to accumulate, but also throughout 237 the cell (Fig. 3D). This meant that the addition of JA induces a certain conformational change to 238 the OsCCR14-OsATL53 complex that enables the two FRET probes to come within the minimum 239 FÖrster distance and cause energy transfer. Such a change in donor lifetime was not observed in 240 the control (OsFBK1-EGFP donor, EYFP-OsCCR14 acceptor) (Fig. S7). Such conformational 241 changes in the OsCCR14-OsATL53 complex might also explain why BiFC fluorescence 242 disappeared after $4 \mathrm{~h}$ of JA exposure (Fig. 3).

243 We had also mentioned that the BiFC interaction between OsCCR14-OsATL53 was stable for long 244 duration post incubation after particle bombardment, but its conformation and localization was 245 affected by JA. Therefore, we wanted to investigate whether JA affects the stability of the 246 OsCCR14-OsATL53 complex in rice. We carried out cell-free degradation of 6xHis-OsCCR14 and $2476 x$ His-OsATL53 in whole protein extracts of WT rice seedlings as described previously (32). Figure $2483 \mathrm{C}$ clearly shows JA influencing the stability of OsCCR14-OsATL53 complex after $4 \mathrm{~h}$ of treatment 249 by hastening the degradation of OSATL53, without affecting OsCCR14 during the experimental 
250 timeframe. However, in absence of JA, OsATL53 is seen to be stable in the cell-free experiments

251 when present as a complex with OsCCR14 (Fig. 3E). This is a stark contrast to the observation

252 where the half-life of OsATL53 has been found to be considerably less when present alone (Fig.

2532 2A). The results of the cell-free experiments have been quantified and graphically represented in

254 Fig. 3F.

255

256

257

258

259

260

Jasmonic acid induces changes in the OsFBK1/OsCCR14-OsATL53 interaction and localization dynamics

As demonstrated above, OsFBK1 interacts with OsCCR14 and OsATL53 individually to mediate their degradation via the $26 \mathrm{~S}$ proteasome pathway in different cellular components. We had also observed that OsATL53 probably enters the nucleus while forming a complex with OsFBK1 (Fig. $1 \mathrm{E}$ and Fig. S6). It was thus, imperative to check the nature of interaction between OsFBK1 and OsCCR14-OsATL53 complex and determine whether JA affects interaction amongst all these proteins. For this, we carried out particle bombardment for a three-way interaction amongst ECFPOsFBK1 and OsCCR14-OsATL53 BiFC. The upper panel in Fig. 4 shows that under normal untreated conditions, OsFBK1 does not seem to overly interact with OsCCR14-OsATL53 complex and follows its normal localization pattern, although some minor interactions could also be seen. However, when the bombarded peels were treated with JA (+MG132) and incubated for $4 \mathrm{~h}, \mathrm{a}$ drastic change in the dynamics of the interactions amongst the three proteins was observed (lower panel, Fig. 4A). The green colour shows the overlapping regions between the ECFP and EYFP fluorescence and it also shows that OsFBK1 interacted with OsCCR14-OsATL53 complex while accumulating around the nucleus, as observed earlier (Fig. 3B). At the same time, in most cells we could also see that OsFBK1 has migrated out of the nucleus into the cytoplasm to interact with the OsCCR14-OsATL53 complex (Fig. 4A and B) which we did not observe for OsFBK1 alone when treated with JA (Fig. S3). Further, we also saw that on exposure to JA (-MG132), there was a loss of EYFP signal in the target cells indicating the disruption of the OsCCR14-OsATL53 complex and the possible degradation of the components in the respective compartments, as also proved by the 
276 cell-free studies (Fig. 4B, Fig. S8). Also, the localization of OsFBK1 also seemed to change back

277 to its original state after this process of degradation is over and its fluorescence appeared

278 throughout the cell and in the nucleus (Fig. 4B). We also noticed some target cells exhibiting faster

279 movement and degradation kinetics, some initiating as sooner as 40 min (+JA, - MG132, Fig.4B)

280 as compared to the cell-free studies (Fig. 3E and F); as expected since the cell-free studies had considerably higher substrate concentration $(5 \mu \mathrm{g})$ than available in native cellular conditions.

282

283

284

285

286

287

288

289

290

291

292

293

294

295

296

297

298

299

300

\section{OsATL53 attenuates enzymatic activity of OsCCR14}

Since OsATL53 forms a stable cytosolic complex with OsCCR14, we investigated whether it affects the enzymatic activity of OsCCR14. OsCCR14 is a cytosolic enzyme and preferentially reduces feruloyl-CoA esters into coniferaldehyde (47). Similarly, SbCCR1 (the sorghum ortholog of OsCCR14) also has a higher affinity for feruloyl-CoA ester as a substrate as opposed to pcoumaroyl CoA and sinapoyl-CoA (48). Thus, we chose feruloyl-CoA as a substrate for investigating the enzymatic activity of OsCCR14 alone, and in complex with OsATL53 (with/without JA). Feruloyl-CoA ester was prepared following the protocol described earlier $(47,48)$ and used as substrate for the reaction mix comprising of: a) OsCCR14, b) OsCCR14 + OsATL53, c) OsATL53, d) OsCCR14 + OsATL53 + JA, and e) OsCCR14 + JA. As evident from Fig. 5A, OsCCR14 shows a $\mathrm{K}_{\mathrm{m}}$ of $6 \mu \mathrm{M}$, whereas with OsATL53 it demonstrates a significant reduction in the substrate affinity $\left(K_{m} 20 \mu \mathrm{M}\right)$. The addition of JA to either OsCCR14 or to the complex, however, does not affect the $K_{m}$ values but slightly affects both their $K_{\text {cat }}$ values (Fig. 5B), thereby signifying a very marginal decrease in enzyme activity.

Our data clearly show that OsATL53 attenuates the enzymatic activity of OsCCR14 by significantly reducing its affinity for feruloyl-CoA, without totally inhibiting its enzymatic activity. We might speculate at this juncture that OsATL53 acts as an allosteric effector molecule rather than an inhibitor and might sequester OsCCR14 in the cytosol as aggregates (as demonstrated by BiFC) to regulate lignification. However, it would require detailed biochemical experimentation to confirm 
301 this assumption. Additionally, the presence of JA does not further reduce the substrate affinity of

302 OsCCR14 as a complex with OsATL53 but changes the conformation of the complex in its

303 presence (Fig. 3) triggering the translocation of the complex towards the nucleus, and for OsFBK1

304 to recognise the complex as a substrate to initiate its degradation via the $26 \mathrm{~S}$ proteasome pathway 305 (Fig. 4).

Rice transgenics of OsCCR14 and OsATL53 demonstrate differential lignin accumulation in roots and anthers

Knock-down rice transgenics of OSATL53 were generated by RNAi, keeping in concert to the OsCCR14KD transgenics reported earlier (32). The downregulation of OsATL53 in these transgenics were analysed by real-time PCR (Fig. S9A). OsCCR14 transcript levels in OsCCR14OE 311 rice transgenics were checked by real-time PCR (Fig. S9C). The roots of 30-day-old WT, 312 OsCCR14 ${ }^{O E}$, OsCCR14 ${ }^{K D}$ and OSATL53 ${ }^{K D}$ transgenics were measured and observed for 313 morphological changes. The roots of OsCCR14 ${ }^{K D}$ were of similar length to WT, although they 314 displayed lesser root density w.r.t. WT (Fig. 6A, upper panel). The roots of OsCCR14OE and 315 OsATL53 ${ }^{K D}$, however, were shorter and less thick as compared to WT (Fig. 6A, middle and bottom 316 panels respectively). The roots lengths are graphically represented in Fig. 6B. Lignin estimation of 317 the same 30-day-old roots of the transgenics and WT showed that OsCCR14 OE and OsATL53 ${ }^{K D}$ 318 accumulated more lignin as compared to WT, while those of OsCCR14 ${ }^{K D}$ had a lesser amount of 319 lignin (Fig. 6C). These results show correlation with the enzyme kinetics data where OsATL53 320 seem to attenuate the enzymatic activity of OsCCR14 in producing lignin.

321 The anthers of OsCCR14 ${ }^{O E}$, OsCCR14 ${ }^{K D}$, OsATL53 ${ }^{K D}$ transgenics and WT were cleared and their 322 lignin visualised as described earlier (32). While the anthers of OsCCR14 ${ }^{K D}$ displayed reduced 323 thickenings as compared to WT, those of OSCCR14OE and OSATL53 ${ }^{K D}$ showed significantly more 324 lignin. The accumulation of $U$-shaped thickenings in the anther bases were more evident than in 325 apices (Fig. 6D). Our data thus proves that OsATL53 regulates the lignification activity of 
326 OsCCR14 in anthers. Additionally, the anthers of OsATL53 ${ }^{K D}$ transgenics are comparatively

327 shorter in length as compared to WT (Fig. S9E).

328 However, when we also checked the expression levels of OsCCR14 in the same OsATL53KD 329 transgenics, we found that OsCCR14 transcript levels were more abundant than WT (Fig. S9B). 330 However, the transcript levels of OSATL53 in OSCCR14OE plants were comparable to WT (Fig. 331 S9D). Therefore, we also believe that OsCCR14 is regulated by OsATL53 even at the transcript 332 level via an unknown mechanism. Whether OsATL53 physically regulates the transcription of 333 OsCCR14 or does so by interacting with other protein partners is being investigated.

\section{DISCUSSION}

335 The ATL family of RING-H2 proteins are a dynamic group that has been studied in the recent 336 years. Several ATL proteins have been explored in Arabidopsis and other plant species and 337 diverse functions have been attributed to them (26), many thought to be functioning as putative 338 RING-H2 type E3 ligases (13). The diversity in the architecture of the ATLs has been proposed to 339 recognise and target substrates in different cellular environments (33). We found OsATL53 to be a 340 component of a putative SCFOsFBK1 and to have auto-ubiquitination properties (Fig. 1), and we also 341 confirmed OsATL53 interacting with the F-box protein OsFBK1 and with its substrate, cinnamoyl 342 CoA-reductase enzyme, OsCCR14 (Fig. 1). However, the cellular localization of these two 343 interactions differed, indicating that OsATL53 interacts with these two proteins differently. These 344 variations in the interactions could be due to the different domains present in OsATL53 as 345 described earlier (33) and have been proved true by deletion studies (Fig. 1).

346 Seeing that both OsCCR14 and OsATL53 are substrates of SCFOsFBK1 and, OsATL53 itself is likely 347 to be a component of the same E3 ligase, it appears that OsATL53 performs other functions apart 348 from participating in the ubiquitination process. OsCCR14 has been found to be the closest 349 homologue to AtCCR1 and has been suggested to be primarily involved in actively lignifying 350 tissues like stems and roots (47). It was also found that OsCCR14 expresses constitutively 
351 throughout the various developmental stages of rice (47). Since OsCCR14 and OsATL53 were

352 both identified as single target clones in the same anther library, we speculate that these two

353 proteins might be together involved in the lignification processes in anthers and in other target

354 tissues. Jasmonic acid has been previously studied extensively for its role in anther dehiscence

$355(39,40,44)$. Additionally, the ubiquitin system and jasmonate signalling pathway are closely linked

where the ubiquitin system contributes to the regulation of the synthesis, perception and signal transduction of JA (49). JA was found to induce conformational changes in an erstwhile stable OsCCR14-OsATL53 complex triggering the accumulation of the complex along the nuclear periphery that initiated their degradation (Fig. 2, 3, 4B, S8). We believe that this translocation could be attributed to OsATL53 (Fig. S3) via an unknown mechanism. RBX1 RING-H2 proteins have been quoted to "function as allosteric activators of E2 enzymes and play an essential role in ubiquitination, although their precise mechanism of action is unknown" $(7,50,51)$, which made us wonder whether plant ATLs might also participate in other enzymatic processes apart from ubiquitination of target substrates. Thus, we provide evidence that the RING-H2/ATL protein, OsATL53, interacts with OsCCR14 and might function to decrease its enzymatic activity.

To account for the role of OsFBK1 in this entire process, we found that in an uninduced state, OsFBK1 does not interact with the OsCCR14-OsATL53 complex but does so with the proteins individually (Fig. 4A). But, as soon as JA is applied, OsFBK1 not only interacts with the OsCCR14OsATL53 complex in the cytoplasm but also travels with the complex towards the nucleus (albeit not appearing to enter the nucleus with the OsCCR14-OsATL53 complex) (Fig. 4A). SCFOsFBk1 371 then mediates the degradation of OsATL53 in the cytoplasm (Fig. 2E and 3E) thereby destabilising 372 the OsCCR14-OsATL53 complex and enabling OsCCR14 and the remaining OsATL53 to enter the 373 nucleus to be degraded via the $26 \mathrm{~S}$ proteasome. We also believe that the JA-induced 374 conformational changes brought about in the OsCCR14-OsATL53 complex (Fig. 3C and D) might 375 be the trigger for the movement and subsequent degradation of the complex by OsFBK1. Such 
conformational changes brought about by JA in proteins and their subsequent degradation by the 26S proteasome have been previously reported for the JAZ degron (52).

Since OsCCR14 catalyses the first limiting step in lignin formation in the cell cytoplasm, our data reveals that OsATL53 slows down the enzymatic processes of OsCCR14, probably sequesters OsCCR14 in the cytosol as aggregates as demonstrated by BiFC, and, thereby, functions to regulate lignification. Microarray data obtained from previously conducted experiments (1) have shown that the expressions of OsCCR14 and OSATL53 overlap in tissues like roots of 7-day-old seedlings, inflorescence stages like P4 and P6 and seed stage S5, whereas their expression differs in the other stages of development examined (Fig. S10). Based on these data, it can be suggested that OsATL53 affects the lignification processes catalysed by OsCCR14 in different target tissues depending on the requirements of that development stage of rice plant. The presence of JA in tissues like late anther stages, however, changes the dynamics of the enzymatic processes and signals the recognition of the OsATL53-OsCCR14 as substrates for destruction via the SCFOsFBK1 E3 ligase. As proof of concept that OsATL53 regulates the activity of OsCCR14, the roots and anthers of OSATL53 ${ }^{K D}$ transgenics display increased lignin thickenings as compared to WT and was comparable to the increased lignification displayed by the anthers and roots of OsCCR $14^{O E}$ transgenics. However, as expected, the roots and anthers of OsCCR $14^{K D}$ transgenics have less lignin (Fig. 6). Surprisingly, OsATL53 also seems to regulate the transcript abundance of OsCCR14 via an unknown pathway that we are currently exploring (Fig. S9C).

Finally, we propose the following model (Fig. 6): During the initial anther development stages where lignification is controlled by auxin, OsCCR14 executes its enzymatic processes in the cytoplasm whereas the increased presence of OsATL53 in the later stages reduces this enzymatic activity. However, towards the later stages of anther maturation preceding dehiscence, when the level of JA starts to increase, the process of lignification needs to cease, and the proteins involved need to be turned over. At this stage, the presence of increasing levels of JA in the cell might 
401 change the conformation of the OsCCR14-OsATL53 complex thereby triggering OsATL53 to travel 402 towards the nucleus, while carrying OsCCR14 along with it as a payload. The change in 403 conformation in the complex is recognised by SCFOsFBK1 as substrates and initiates the degradation 404 of OsATL53, thereby disassociating the complex and enabling OsCCR14 to enter the nucleus 405 where it is finally degraded by the $26 \mathrm{~S}$ proteasome. Also, some of the remaining OsATL53 might 406 enter the nucleus as an OsFBK1-OsATL53 complex where OsATL53 would also be finally 407 degraded. OsATL53 might also regulate the transcription of OsCCR14 by an unknown pathway.

408 To summarise, we have reported a divergent role of ATL RING-H2 proteins in interacting with 409 other proteins, regulating their activities, and in turn become substrates of E3 ligases, thereby 410 highlighting the diversity in their functions apart from participating as a bridging partner between 411 the E3 and E2 ligases. Additionally, the modulation of OsCCR14 enzymatic function by OsATL53 412 could have application in terms of manipulating the lignin content in cash crops. At the same time, 413 we provide an insight into the functioning of the F-box protein, OsFBK1, in JA-induced targeted 414 substrate degradation that would in turn regulate anther development in rice with precision.

\section{METHODS}

\section{Gene constructs}

417 The 609 bp of the OsATL53 gene (LOC_Os04g48310) was cloned in the following vectors: 418 pET28a (EcoRI/Sacl) (Novagen); pGEX4T1 (EcoRI/Sall) (GE); pENTR-D-TOPO (5' CACC 419 overhang) entry vector and BiFC Gateway ${ }^{\mathrm{TM}}$ vectors, pSITE-3CA-EYFPC ${ }_{1}$ and pSITE-3CA420 EYFPN $_{1}$ using Gateway ${ }^{\mathrm{TM}}$ technology (pENTR ${ }^{\mathrm{TM}}$ Directional TOPO ${ }^{\circledR}$ cloning kit, and LR clonase 421 Enzyme mix II kit; Invitrogen Inc. USA). CUL5g (LOC_Os05g05700) was cloned in pGEX4T1 422 vector $(B a m H I / S a l l)$. The deleted constructs of OsATL53 (510 bp of $\triangle O$ OsATL53, 411 bp of 423 OsATL53 4 ) were cloned in the pGEX4T1 vector (EcoRl/Xhol). The cloning of OsFBK1 424 (LOC_Os01g47050) and OsCCR14 (LOC_Os08g34280), in their respective vectors has been 425 described earlier (32). For localization and FLIM-FRET experiments, pSITE-1CA/pCAMBIA1302 
426 was used for the donor constructs and pSITE-3CA for the acceptor constructs. Additionally,

427 OsFBK1 was cloned in the pSITE-1CA vector for CFP fluorescence by Gateway ${ }^{\mathrm{TM}}$ cloning. The 428 amplification of the genes was carried out using Phusion ${ }^{\mathrm{TM}}$ high-fidelity Taq polymerase 429 (Finnzymes, Finland) as per manufacturer's instructions (see Table S1 for primers).

\section{Particle bombardment}

Biolistics of the various constructs on onion epidermal peel cells for BiFC, intercellular localization and hormone assays were carried out using Biolistic PDS-1000/He particle delivery system (BioRad, USA) as described earlier (32). For hormone-related visualisation, the bombarded and incubated onion peels $(12-16 \mathrm{~h})$ were treated with $6.4 \mathrm{mM} \mathrm{JA}$ for the requisite time-points before observing for fluorescence under confocal microscope (Leica TCS, SP5). For FLIM-FRET experiments, all observations and analyses were carried out using the Leica SP8 FALCON confocal microscope at the Central Instrumentation Facility (CIF), University of Delhi South Campus. FLIM was carried out at $40 \mathrm{MHz}$ and curve-fitting was carried out using the $\mathrm{n}$ Exponential Reconvolution fit model. For all experiments, the tissue was mounted in water and observed at 20X magnification. All experiments were carried out at least five times, with an average of 3 cells being visualised in each repetition.

\section{Protein induction, western blotting and immunoprecipitation}

Induction and western blotting of 6xHis-OsATL53, 6xHis-OsCCR14, GST-OsATL53, GST-OsFBK1 and GST-Cul5g was carried out as described earlier (32). Immunoprecipitation assays were performed using $100 \mu \mathrm{g}$ of unpurified bait and prey protein lysates as per the protocol described earlier (32). Pre-stained markers used for all blots were Puregene NEX-GEN-PinkADD pre-stained protein ladder (Cat. \# PG500-0500PI, Genetix Asia Pvt. Ltd., New Delhi, India). Western blotting was carried out with anti-His (Cat. \# H1029-5ML, Sigma, USA) and anti-GST (Cat. \# G7781-2ML, Sigma, USA) antibodies.

\section{Auto-ubiquitination assay}


451 The auto-ubiquitination activity of OsATL53 was carried out following a modified protocol described

452 by (25). An E1 and E2 ligase mix (Cat. \# U8382, Sigma, USA) was used for the activation and

453 conjugation of ubiquitin. Negative controls used were generated in the laboratory. Probing of 454 ubiquitin was done using monoclonal anti-ubiquitin antibodies (Cat. \# U0508-50UL, Sigma, USA) 455 at a dilution of $1: 8,000+1 \%$ BSA.

\section{Cell-free degradation}

457 For cell-free degradation assays, total plant protein was extracted from 7-day-old seedlings of WT 458 and OsFBK1 transgenics and cell-free experiments were carried out using $5 \mu \mathrm{g} 6 \mathrm{xHis-OsATL53/5}$ $459 \mu \mathrm{g}$ 6xHis-OsCCR14 in $30 \mu \mathrm{g}$ total plant protein extracts/nuclear-enriched protein 460 extracts/cytoplasmic protein extracts by following the protocol described before (32). Nuclear 461 protein extracts from 10-day-old seedlings (5 $\mathrm{g}$ tissue) were obtained as per protocol described by 462 (53). The cytoplasmic extracts were aliquoted after filtration and the first centrifugation step. For 463 loading controls, the same blots were stained with Ponceau for the detection of the Rubisco protein 464 large subunit band for cell-free with total plant protein and cytoplasmic extracts whereas for cell465 free with the nuclear-enriched protein extracts, Ponceau stained histone H3 band ( 15kDa) was 466 used as loading control. For cell-free experiments with JA, the cell-free degradation cocktail was 467 mixed with $6.4 \mathrm{mM} \mathrm{JA}$ and incubated at $30{ }^{\circ} \mathrm{C}$ for the requisite time points. The degradation data 468 was graphically represented by measuring the intensities of the bands of all blots in Image 469 Studio ${ }^{T M}$ Lite ver. 5.2 (https://www.licor.com/bio/products/software/image_studio_lite/). The $2 \mathrm{~h} / 6 \mathrm{~h}$ 470 western band for each blot of OsATL53 and OsCCR14, respectively, was used as the reference 471 and measured intensities were normalised against it and multiplied by 100. Representation of the 472 data is in percentage intensity ratio. All experiments were carried out at least thrice and error bars 473 denote standard deviation across replicates.

\section{Synthesis of feruloyl-CoA thioester and purification}


475 The protocol for synthesis of the substrate feruloyl-CoA thioester was modified from the processes

476 described earlier $(47,48)$. Briefly, the Arabidopsis 4-coumarate: CoA ligase 1 gene, At4CL1, was

477 amplified from leaf cDNA and cloned in pET28a vector (see Table S1 for primers). The clone was

478 transformed in BL21-RIL competent cells and the protein induced and purified as described earlier 479 (32). For the synthesis of the thioester, $410 \mu \mathrm{g}$ of purified At4CL1 protein was added to a $40 \mathrm{~mL}$ 480 solution containing $50 \mathrm{mM}$ sodium phosphate buffer, $\mathrm{pH}$ 7.0, $800 \mu \mathrm{M}$ CoA (Cat. \# C4282, Sigma, supernatant was dried under vacuum centrifugation. The obtained pellet/substrate was stored at $20{ }^{\circ} \mathrm{C}$ for later use. A standard curve of the obtained ester was generated using $A_{366}$ for measurement using a spectrophotometer.

\section{Enzyme kinetic assay of OsCCR14}

490 The activity of OsCCR14 was measured according to the protocols described $(47,48)$. To a final 491 volume of $210 \mu \mathrm{L}$ of each reaction mixture consisting of $100 \mathrm{mM}$ sodium phosphate buffer, $\mathrm{pH} 6.2$, 0.25 mM NADPH (Cat. \# 144935, SRL Pvt. Ltd., India), thioester substrate (5 $\mu \mathrm{M}, 10 \mu \mathrm{M}, 20 \mu \mathrm{M}$, $50 \mu \mathrm{M}$ and $100 \mu \mathrm{M}), 3 \mu \mathrm{g}$ purified OsCCR14 was added, and the enzyme reactions carried out at $30^{\circ} \mathrm{C}$. For reactions with OsCCR14 and OsATL53 complex, equal amounts of OsCCR14 and OsATL53 were first incubated at $4{ }^{\circ} \mathrm{C}$ for at least $2 \mathrm{~h}$, quantified and $3 \mu \mathrm{g}$ used per reaction. For reactions with $\mathrm{JA}, 100 \mu \mathrm{M} \mathrm{JA}$ was added to each reaction and incubated at $4{ }^{\circ} \mathrm{C}$ for $3 \mathrm{~h}$. Reactions were initiated by the addition of the enzyme for $20 \mathrm{~min}$ with an interval of $1 \mathrm{~min}$ between reactions (20 reactions for one substrate concentration per reaction condition). Reactions were stopped by the addition of $40 \mu \mathrm{L}$ of $15 \%(\mathrm{w} / \mathrm{v})$ trichloroacetic acid and mixed well. These were then centrifuged 
$501 \quad A_{366}$ (substrate concentration) was recorded using a Tecan M200 microplate reader (Tecan Austria

$502 \mathrm{GmbH}$ ) and the $\mathrm{i}$-control 1.7 software (Tecan Austria $\mathrm{GmbH}$ ). $\mathrm{K}_{\mathrm{m}}, \mathrm{V}_{\max }$ and $\mathrm{K}_{\text {cat }}$ values were 503 determined by extrapolation from Lineweaver-Burk plots. Each enzyme assay was carried out for 504 five times and the average values represented the mean \pm standard deviation.

\section{Transgenic analyses}

506 OsATL53 ${ }^{K D}$ and OsCCR14OE transgenic lines were generated in Pusa Basmati 1 by RNAi and 507 overexpression respectively, and their expression levels quantified by real-time PCR as described 508 earlier (32). Lignin estimation of roots of 30-day old seedlings were carried out as described earlier 509 (32). Pre-dehiscent anthers were harvested from at least 3 lines, cleared in lactic acid and 510 visualised using confocal microscopy (Leica SP8 FALCON) as earlier (32). See Table S1 for 511 primers.

512 Author contributions: P.B. designed the experiments and wrote the paper. P.B. and A.S. carried 513 out the experiments. J.P.K. supervised the work and critically edited the article.

\section{SUPPORTING INFORMATION}

515 Fig. S1. Structural analyses of OsATL53.

$516 \quad$ Fig. S2. Predicted putative protein complexes.

517 Fig. S3. Sub-cellular localization of proteins under control and JA treatments.

518 Fig. S4. Sub-cellular localization of OsCCR14+OsATL53 BiFC complexes under control and JA 519 treatments with nuclear stained cells.

520 Fig. S5. Sub-cellular localization of OsATL53 under different JA concentrations.

521 Fig. S6. Sub-cellular co-localization of OsFBK1-OsATL53 under control and JA treatments.

522 Fig. S7. Additional FLIM-FRET data.

523 Fig. S8. Time-lapse confocal images depicting the targeted degradation of EYFP-OSATL53 in the 524 presence of JA.

525 Fig, S9. Real-time expression analyses. 
526 Fig. S10. Microarray expression data of OsCCR14 and OsATL53 at different stages of rice

527 development.

528 Table S1. Primers used in the study.

529 Materials and Methods

530

531

532

533

534

535

536

537

538

539

540

541

542

543

544

545

546

547

548

549

550

551

552

553

554

555

556

\section{REFERENCES}

1. M. Jain et al., F-box proteins in rice. Genome-wide analysis, classification, temporal and spatial gene expression during panicle and seed development, and regulation by light and abiotic stress. Plant Physiol 143, 1467-1483 (2007).

2. Z. Hua, C. Zou, S. H. Shiu, R. D. Vierstra, Phylogenetic comparison of F-Box (FBX) gene superfamily within the plant kingdom reveals divergent evolutionary histories indicative of genomic drift. PLoS One 6, e16219 (2011).

3. R. D. Vierstra, The ubiquitin-26S proteasome system at the nexus of plant biology. Nat Rev Mol Cell Biol 10, 385-397 (2009).

4. L. Buetow, D. T. Huang, Structural insights into the catalysis and regulation of E3 ubiquitin ligases. Nat Rev Mol Cell Biol 17, 626-642 (2016).

5. Z. Hua, R. D. Vierstra, The cullin-RING ubiquitin-protein ligases. Annu Rev Plant Biol 62 , 299-334 (2011).

6. P. S. Freemont, I. M. Hanson, J. Trowsdale, A novel cysteine-rich sequence motif. Cell 64, 483-484 (1991).

7. P. K. Jackson et al., The lore of the RINGs: substrate recognition and catalysis by ubiquitin ligases. Trends Cell Biol 10, 429-439 (2000).

8. D. Jiménez-López, F. Muñóz-Belman, J. M. González-Prieto, V. Aguilar-Hernández, P. Guzmán, Repertoire of plant RING E3 ubiquitin ligases revisited: New groups counting gene families and single genes. PLoS One 13, e0203442 (2018).

9. S. D. Lim et al., A gene family encoding RING finger proteins in rice: their expansion, expression diversity, and co-expressed genes. Plant Mol Biol 72, 369-380 (2010).

10. I. Alam et al., Genome-wide identification, evolution and expression analysis of RING finger protein genes in Brassica rapa. Sci Rep 7, 40690 (2017).

11. R. E. Salinas-Mondragón, C. Garcidueñas-Piña, P. Guzmán, Early elicitor induction in members of a novel multigene family coding for highly related RING-H2 proteins in Arabidopsis thaliana. Plant Mol Biol 40, 579-590 (1999). 
12. M. Serrano, P. Guzmán, Isolation and gene expression analysis of Arabidopsis thaliana mutants with constitutive expression of ATL2, an early elicitor-response RING-H2 zincfinger gene. Genetics 167, 919-929 (2004).

13. M. Serrano, S. Parra, L. D. Alcaraz, P. Guzmán, The ATL gene family from Arabidopsis thaliana and Oryza sativa comprises a large number of putative ubiquitin ligases of the RING-H2 type. J Mol Evol 62, 434-445 (2006).

14. P. Guzmán, ATLs and BTLs, plant-specific and general eukaryotic structurally-related E3 ubiquitin ligases. Plant Sci 215-216, 69-75 (2014).

15. D. Jiménez-López, L. Aguilar-Henonin, J. M. González-Prieto, V. Aguilar-Hernández, P. Guzmán, CTLs, a new class of RING-H2 ubiquitin ligases uncovered by YEELL, a motif close to the RING domain that is present across eukaryotes. PLoS One 13, e0190969 (2018).

16. X. W. Deng et al., COP1, an Arabidopsis regulatory gene, encodes a protein with both a zinc-binding motif and a G beta homologous domain. Cell 71, 791-801 (1992).

17. C. S. Hardtke, H. Okamoto, C. Stoop-Myer, X. W. Deng, Biochemical evidence for ubiquitin ligase activity of the Arabidopsis COP1 interacting protein 8 (CIP8). Plant $J$ 30, 385-394 (2002).

18. Q. Xie et al., SINAT5 promotes ubiquitin-related degradation of NAC1 to attenuate auxin signals. Nature 419, 167-170 (2002).

19. R. Xu, Q. Q. Li, A RING-H2 zinc-finger protein gene RIE1 is essential for seed development in Arabidopsis. Plant Mol Biol 53, 37-50 (2003).

20. C. H. Dong, M. Agarwal, Y. Zhang, Q. Xie, J. K. Zhu, The negative regulator of plant cold responses, HOS1, is a RING E3 ligase that mediates the ubiquitination and degradation of ICE1. Proc Natl Acad Sci U S A 103, 8281-8286 (2006).

21. S. L. Stone, E. M. Anderson, R. T. Mullen, D. R. Goring, ARC1 is an E3 ubiquitin ligase and promotes the ubiquitination of proteins during the rejection of self-incompatible Brassica pollen. Plant Cell 15, 885-898 (2003).

22. Y. Sonoda et al., SHA1, a novel RING finger protein, functions in shoot apical meristem maintenance in Arabidopsis. Plant J 50, 586-596 (2007).

23. X. Zhang, V. Garreton, N. H. Chua, The AIP2 E3 ligase acts as a novel negative regulator of ABA signaling by promoting ABI3 degradation. Genes Dev 19, 1532-1543 (2005).

24. J. Liu et al., Targeted degradation of the cyclin-dependent kinase inhibitor ICK4/KRP6 by RING-type E3 ligases is essential for mitotic cell cycle progression during Arabidopsis gametogenesis. Plant Cell 20, 1538-1554 (2008). 
591 25. Y. J. Peng et al., A RING-type E3 ligase controls anther dehiscence by activating the jasmonate biosynthetic pathway gene DEFECTIVE IN ANTHER DEHISCENCE1 in Arabidopsis. Plant J 74, 310-327 (2013).

594 26. P. Guzmán, The prolific ATL family of RING-H2 ubiquitin ligases. Plant Signal Behav 7, 1014-1021 (2012).

27. S. Disch et al., The E3 ubiquitin ligase BIG BROTHER controls arabidopsis organ size in a

28. S. L. Poulsen et al., RNF111/Arkadia is a SUMO-targeted ubiquitin ligase that facilitates the DNA damage response. J Cell Biol 201, 797-807 (2013).

600

29. C. E. Kelly et al., Rnf165/Ark2C enhances BMP-Smad signaling to mediate motor axon

601

602 extension. PLoS Biol 11, e1001538 (2013).

603

604

605

606

607

608

609

610

611

612

613

614

615

616

617

618

619

620

621

30. J. E. Sheren, C. K. Kassenbrock, RNF38 encodes a nuclear ubiquitin protein ligase that modifies p53. Biochem Biophys Res Commun 440, 473-478 (2013).

31. T. Kawasaki et al., Cinnamoyl-CoA reductase, a key enzyme in lignin biosynthesis, is an effector of small GTPase Rac in defense signaling in rice. Proc Natl Acad Sci U S A 103, 230-235 (2006).

32. P. Borah, J. P. Khurana, The OsFBK1 E3 Ligase Subunit Affects Anther and Root Secondary Cell Wall Thickenings by Mediating Turnover of a Cinnamoyl-CoA Reductase. Plant Physiol 176, 2148-2165 (2018).

33. V. Aguilar-Hernández, L. Aguilar-Henonin, P. Guzmán, Diversity in the architecture of ATLs, a family of plant ubiquitin-ligases, leads to recognition and targeting of substrates in different cellular environments. PLoS One 6, e23934 (2011).

34. M. Furukawa, Y. Zhang, J. McCarville, T. Ohta, Y. Xiong, The CUL1 C-terminal sequence and ROC1 are required for efficient nuclear accumulation, NEDD8 modification, and ubiquitin ligase activity of CUL1. Mol Cell Biol 20, 8185-8197 (2000).

35. K. Greve, T. La Cour, M. K. Jensen, F. M. Poulsen, K. Skriver, Interactions between plant RING-H2 and plant-specific NAC (NAM/ATAF1/2/CUC2) proteins: RING-H2 molecular specificity and cellular localization. Biochem J 371, 97-108 (2003).

36. V. D'Angiolella et al., Cyclin F-mediated degradation of ribonucleotide reductase M2 controls genome integrity and DNA repair. Cell 149, 1023-1034 (2012).

622 practical applications. Plant Cell 5, 1217-1229 (1993). 
38. V. Cecchetti, M. M. Altamura, G. Falasca, P. Costantino, M. Cardarelli, Auxin regulates Arabidopsis anther dehiscence, pollen maturation, and filament elongation. Plant Cell 20, 1760-1774 (2008).

39. P. M. Sanders et al., The arabidopsis DELAYED DEHISCENCE1 gene encodes an enzyme in the jasmonic acid synthesis pathway. Plant Cell 12, 1041-1061 (2000).

40. S. Ishiguro, A. Kawai-Oda, J. Ueda, I. Nishida, K. Okada, The DEFECTIVE IN ANTHER DEHISCIENCE gene encodes a novel phospholipase A1 catalyzing the initial step of jasmonic acid biosynthesis, which synchronizes pollen maturation, anther dehiscence, and flower opening in Arabidopsis. Plant Cell 13, 2191-2209 (2001).

41. D. X. Xie, B. F. Feys, S. James, M. Nieto-Rostro, J. G. Turner, COI1: an Arabidopsis gene required for jasmonate-regulated defense and fertility. Science 280, 1091-1094 (1998).

42. A. Stintzi, J. Browse, The Arabidopsis male-sterile mutant, opr3, lacks the 12oxophytodienoic acid reductase required for jasmonate synthesis. Proc Natl Acad Sci U S A 97, 10625-10630 (2000).

43. A. Devoto et al., COI1 links jasmonate signalling and fertility to the SCF ubiquitin-ligase complex in Arabidopsis. Plant J 32, 457-466 (2002).

44. V. Cecchetti et al., Auxin controls Arabidopsis anther dehiscence by regulating endothecium lignification and jasmonic acid biosynthesis. Plant J 74, 411-422 (2013).

45. S. Trautmann et al., "Fluorescence lifetime imaging (FLIM) in confocal microscopy applications: an overview," (2013).

46. T. J. Lambert, FPbase: a community-editable fluorescent protein database. Nat Methods 16, 277-278 (2019).

47. H. L. Park, S. H. Bhoo, M. Kwon, S. W. Lee, M. H. Cho, Biochemical and Expression Analyses of the Rice Cinnamoyl-CoA Reductase Gene Family. Front Plant Sci 8, 2099 (2017).

48. S. A. Sattler, A. M. Walker, W. Vermerris, S. E. Sattler, C. Kang, Structural and Biochemical Characterization of Cinnamoyl-CoA Reductases. Plant Physiol 173, 10311044 (2017).

49. A. Nagels Durand, L. Pauwels, A. Goossens, The Ubiquitin System and Jasmonate Signaling. Plants (Basel) 5, (2016).

50. P. S. Freemont, RING for destruction? Curr Biol 10, R84-87 (2000).

51. M. A. Noureddine, T. D. Donaldson, S. A. Thacker, R. J. Duronio, Drosophila Roc1a encodes a RING-H2 protein with a unique function in processing the Hh signal transducer Ci by the SCF E3 ubiquitin ligase. Dev Cell 2, 757-770 (2002). 
657 52. L. B. Sheard et al., Jasmonate perception by inositol-phosphate-potentiated COI1-JAZ co-

658

659

660

661

662

663

664

665

666

667

668

669

670

671

672

673

674

675

676

677

678

679

680

681

682

683 receptor. Nature 468, 400-405 (2010).

53. F. Xu, S. Xu, M. Wiermer, Y. Zhang, X. Li, The cyclin L homolog MOS12 and the MOS4associated complex are required for the proper splicing of plant resistance genes. Plant $J$ 70, 916-928 (2012).

\section{FIGURE LEGENDS}

Fig. 1. Interaction studies of OsATL53. A. In vitro immunoprecipitation assay between GST-CUL5g and 6xHis-OsATL53. Lanes 2, 3 and 4 are the unpurified input bacterial extracts of GST, GSTCUL5g and 6xHis-OsATL53, respectively. GST wash was used as a negative control for IP with 6xHis-OsFBK1 (lane 5). GST-tagged proteins were probed by rabbit anti-GST mAb, while 6xHisOsATL53 was detected by using mouse anti-His mAb. These blots were parallelly processed with the same samples. M - Marker lane with the designated protein bands. B. In vitro auto-ubiquitintion assay to determine E3 property of OsATL53. Controls used are GST-OsATL53 4 and GST$\triangle$ OsATL53 respectively. Arrows determine the positions of ubiquitinated OsATL53/GST-OsATL53 in top panel, red asterisks indicate non-specific bands. Bottom panel shows the input proteins as probed by anti-GST mAb. C. Representative image of cellular localization of OsATL53 in onion epidermal peel cells. Control: pSITE-3CA vector. Bright field images show the location of the nuclei in all panels. Bar- $50 \mu \mathrm{m}$. Red boxes denote nucleus. D. In vitro immunoprecipitation assay between GST-OsFBK1 and 6xHis-OsATL53. GST wash (lane 2) was used as the negative control. Lanes 3 and 4 show the input bacterial extracts of GST-OsFBK1 and 6xHis-OsATL53 respectively. Lanes 5 and 6 are the IP lanes. E. YFP fluorescence showing the BiFC between OsATL53/OsFBK1 (upper panel) and OsATL53/OsCCR14 (middle panel) respectively in a representative cell. Bright field images show the location of the nuclei. Negative control: BiFC between OsATL53 and EFTu (lower panel). Bar- $50 \mu \mathrm{m}$. Red boxes denote nucleus. F. IP between GST-OsATL53 and 6xHisOsCCR14. G. Western blot showing the IPs between GST- $\Delta$ OsATL53/ GST-OsATL53 $\Delta$ and OsCCR14 and OsFBK1 respectively. Red asterisks denote non-specific binding. For all IPs, GST 
and GST-tagged proteins were probed by rabbit anti-GST mAb, His-tagged proteins were detected by mouse anti-His mAb. M- marker lane for both blots. See also Fig. S1.

Fig. 2. Cell-free degradation of OsATL53 in plant protein extracts. A. Time-scale degradation assay of $5 \mu \mathrm{g}$ 6xHis-OsATL53 in $30 \mu \mathrm{g}$ cell-free whole seedling protein extracts of control and OsFBK1 transgenics both with and without MG132. Loading control depicted by the Ponceau stained Rubisco large subunit band (55 kDa) for all. The bottom-most panel shows the level of OsFBK1 in the plant materials used, as probed by anti-OsFBK1 antibodies described earlier (Borah and Khurana, 2018). B. Graphical representation of degradation kinetics data of OsATL53. Error bars denote SD. C. Degradation assay of $6 x$ His-OsCCR14 in the nuclear protein-enriched and cytoplasmic protein extracts of WT and OsFBK1OE seedlings without MG132. The time points used were as described before (Borah and Khurana, 2018). The confocal image on the left shows the BiFC interaction between OsFBK1 and OsCCR14 in onion peel cells. Loading controls are Ponceau stained Rubisco (55 kDa) for cytoplasmic extracts and histone H3 band (15 kDa) for nuclear protein-enriched extracts respectively. D. Graphical representation of the same where error bars denote SD. E. Cell-free degradation time kinetics of 6xHis-OsATL53 in the nuclear protein-enriched and cytoplasmic protein extracts of WT and OsFBK1OE seedlings without MG132. Image of BiFC between OsFBK1 and OsATL53 in onion epidermal peel cells is shown on the left. Loading controls, histone $\mathrm{H} 3$ and Rubisco large subunit as mentioned above. F. Percentage intensity ratio graph of OsATL53 degradation in the cell-free systems. Error bars denote SD.

Fig. 3. Jasmonic acid induced changes in sub-cellular location and conformation of OsATL53OsCCR14 complex. A. Co-localization of OsCCR14-ECFP and OsATL53-EYFP in onion peel cells. Upper panel shows ECFP and EYFP fluorescence without JA treatment, while lower panel shows changes in EYFP fluorescence after 4 hours of JA treatment. Red boxes show the accumulation of ECFP and EYFP fluorescence around the target cell's nucleus before and after JA treatment. $\mathbf{B}$. Movement of OsATL53-OsCCR14 BiFC complex in response to $6.4 \mathrm{mM}$ JA observed after 4 hours. 
Bright field images show the location of the nucleus in the representative target cell. Bar- $50 \mu \mathrm{m}$. C.

710 Change in lifetime of ECFP-OsCCR14 donor construct upon exposure to JA under FLIM-FRET

711 conditions. Error bars denote SD of at least 5 cells observed. D. Representative Fast FLIM images

712 of target cells in normal and JA-induced conditions. Positive FRET is present through-out the cell.

713 Bar represents the range of lifetimes from 0.5-3.5 ns. E. Cell-free degradation of $6 x H i s-O s C C R 14$

714 and 6xHis-OsATL53 in the cytoplasmic protein extracts of WT seedlings, in the absence and

715 presence of JA respectively. Time kinetics performed in the absence of MG132. Loading control:

716 Ponceau stained Rubisco large subunit band (55 kDa). Red arrows denote non-specific binding. M -

717 marker lane. F. Graphical representation of cell-free degradation kinetics where error bars denote

718 SD. See Fig. S3-S6.

719 Fig. 4. Changes in protein interaction and localization dynamics in the presence of JA. A. OsFBK1 720 ECFP fluorescence was observed to merge with the BiFC (OsATL53-OsCCR14) EYFP 721 fluorescence (Z-stacked) after 2 hours of JA exposure and accumulate around the nuclear 722 boundary after 4 hours, in the presence of MG132. B. Changes in localization pattern of OsFBK1 723 after JA without MG132 treatment in presence of OsCCR14-OsATL53 complex. After degradation 724 of OsATL53 and the disruption of the OsCCR14-OsATL53 complex and loss of EYFP signal, the 725 normal localization pattern of OsFBK1 is seen. The same target cell was observed after the 726 requisite time points after JA treatment. A loss in signal strength was observed due to repeated 727 laser exposure, and the ECFP and EYFP fluorescence were adjusted accordingly. The orange 728 boxes and inset pictures below each panel focusses the nucleus. Bright field images show the 729 location of the nucleus in each target cell. Merged pictures are shown in both dark and bright field 730 background. Green colour denotes overlapped regions of ECFP and EYFP fluorescence. Bar - 50 $731 \mu \mathrm{m}$. See Fig. S7.

732 Fig. 5. A: Kinetic parameters of the enzymatic activities of OsCCR14 under different conditions. 733 Graphical representation of the $\mathrm{K}_{\mathrm{m}}$ values of OsCCR14 either alone or in a complex with OsATL53, 
734 with or without JA. B: Tabular representation of the observed kinetic parameters of the various 735 enzyme combinations.

736 Fig. 6. Transgenic morphological data. A: Root morphology of 30-day-old seedlings. B: Graphical 737 representation of root length of 30-day-old seedlings. Error bars - SD. C: Lignin estimation in roots 738 of 30-day-old seedlings. Error bars - SD. D: Observation of wall morphology in representative rice 739 pre-dehiscent anthers of wild type and transgenics. Confocal microscopy of cleared anther apices 740 (top panels) and bases (bottom panels) of OsCCR $14^{K D}$ and OSATL53 ${ }^{K D}$ transgenics and wild type 741 showing the differences in the dispersal of the U-shaped wall thickenings in the endothecium. The 742 U-shaped thickenings are indicated by arrows. Bar $=50 \mu \mathrm{m}$. Asterisk denotes separate locules of a 743 single anther in the same frame. The globular structures are pollen grains.

744 Fig. 7. Proposed model of the signalling pathway involved in the jasmonic acid-mediated 745 degradation of OsATL53 and OsCCR14 by SCF ${ }^{\text {OsFBK1 } 1}$ and the $26 \mathrm{~S}$ proteasome. OsFBK1 interacting 746 with OSK1/OSK20 has been reported earlier (Borah and Khurana, 2018). See text for more details. 


\section{$\mathbf{A}$}

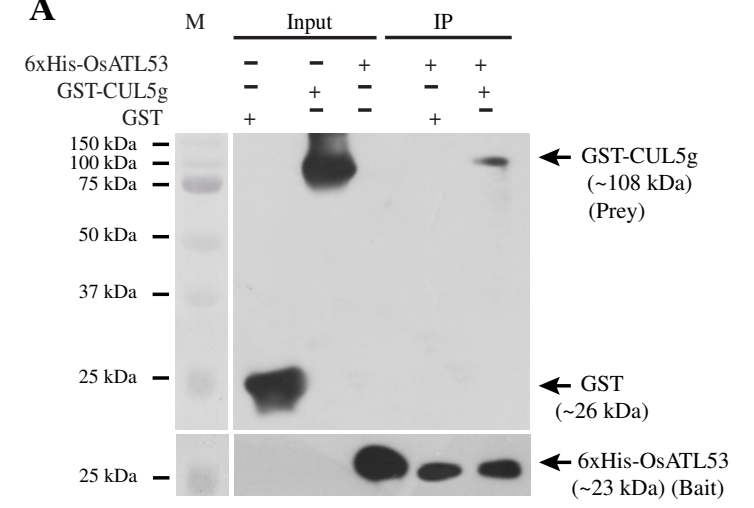

\section{D}

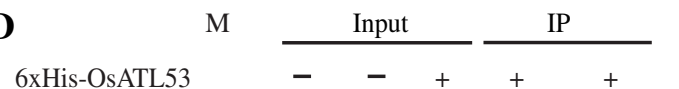

GST-OsFBL53

GST-OsFBK1

GST

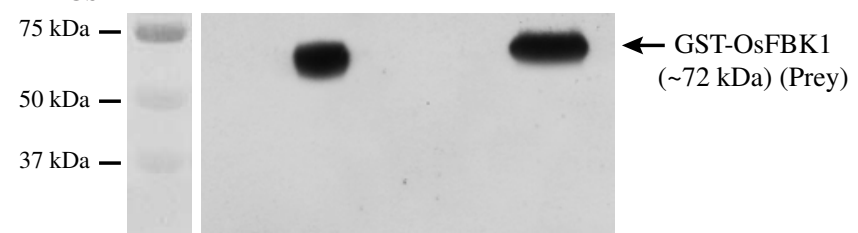

$25 \mathrm{kDa}$

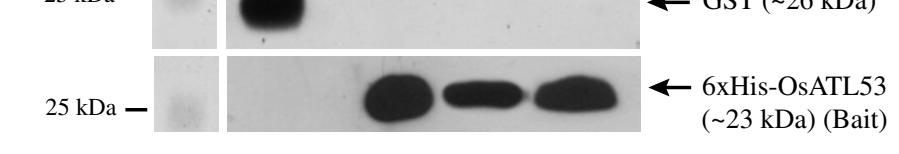

$\mathbf{F}$

6xHis-OsCCR 14

GST-OsATL53

GST

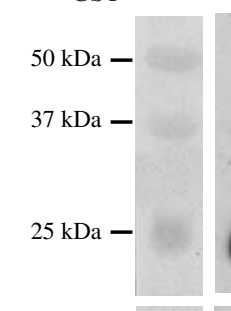

$50 \mathrm{kDa}-$

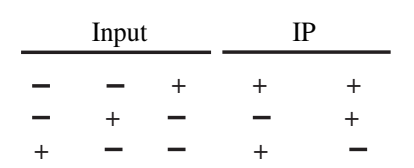

( $23 \mathrm{kDa})$ (Bait)

G

6xHis-OsCCR14
6xHis-OsBRK1
GST-OSATL53
GST- - OsATL53

GST
$50 \mathrm{kDa}-$
$37 \mathrm{kDa}-$
$25 \mathrm{kDa}-$

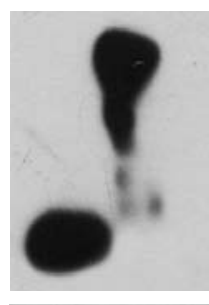

$\longrightarrow \longrightarrow \underset{(\sim 45 \text { kDa) (Bait) }}{6 x \mathrm{His}-\mathrm{OsCCR} 14}$

$\leftarrow$ GST-OsATL53

$(\sim 47 \mathrm{kDa})(\mathrm{Prey})$

GST ( $26 \mathrm{kDa})$

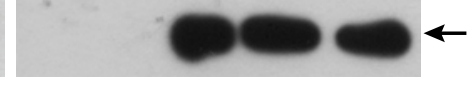

IP

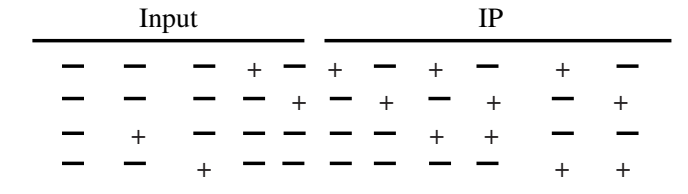

B $\mathrm{E} 1+\mathrm{E} 2++-+++\mathrm{M}$
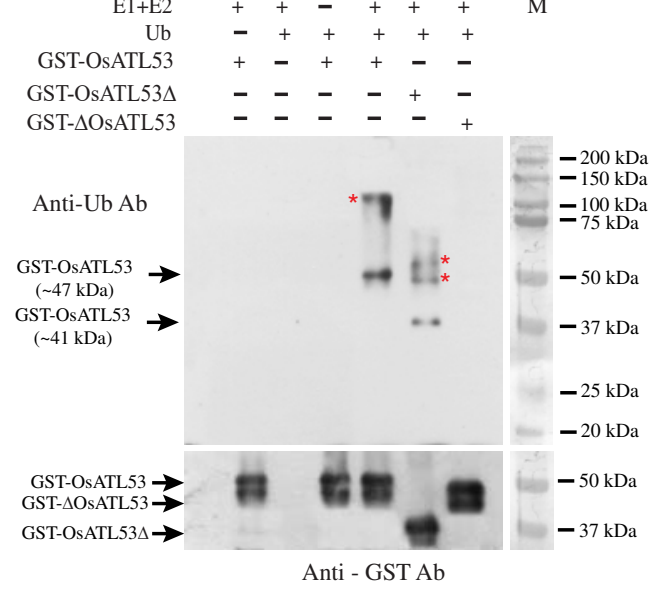

C Fluorescence Bright field $\quad$ Merged

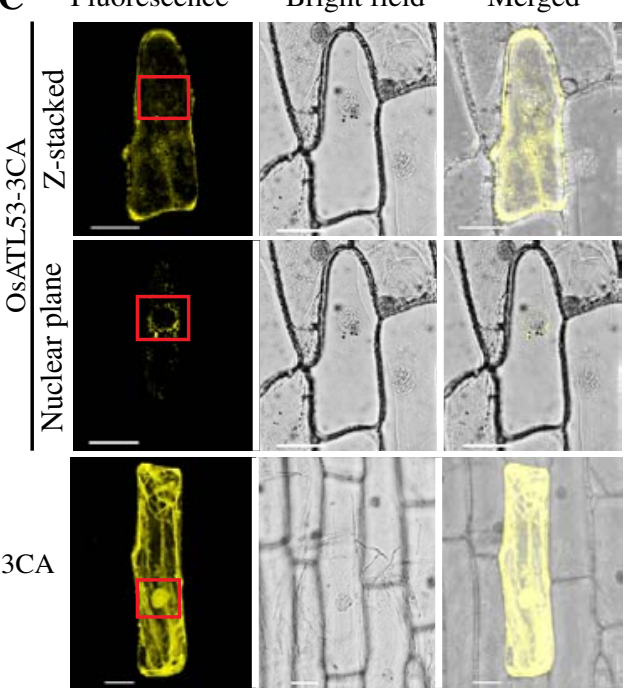

$\mathbf{E}$

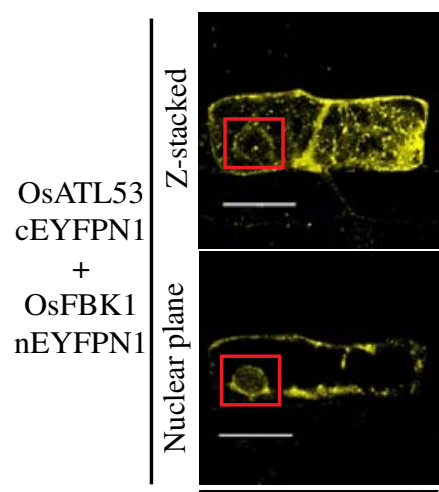

OsATL53

cEYFPN1

$\stackrel{+}{+}$

nEYFPC1

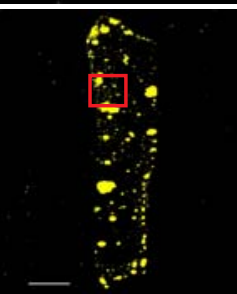

7
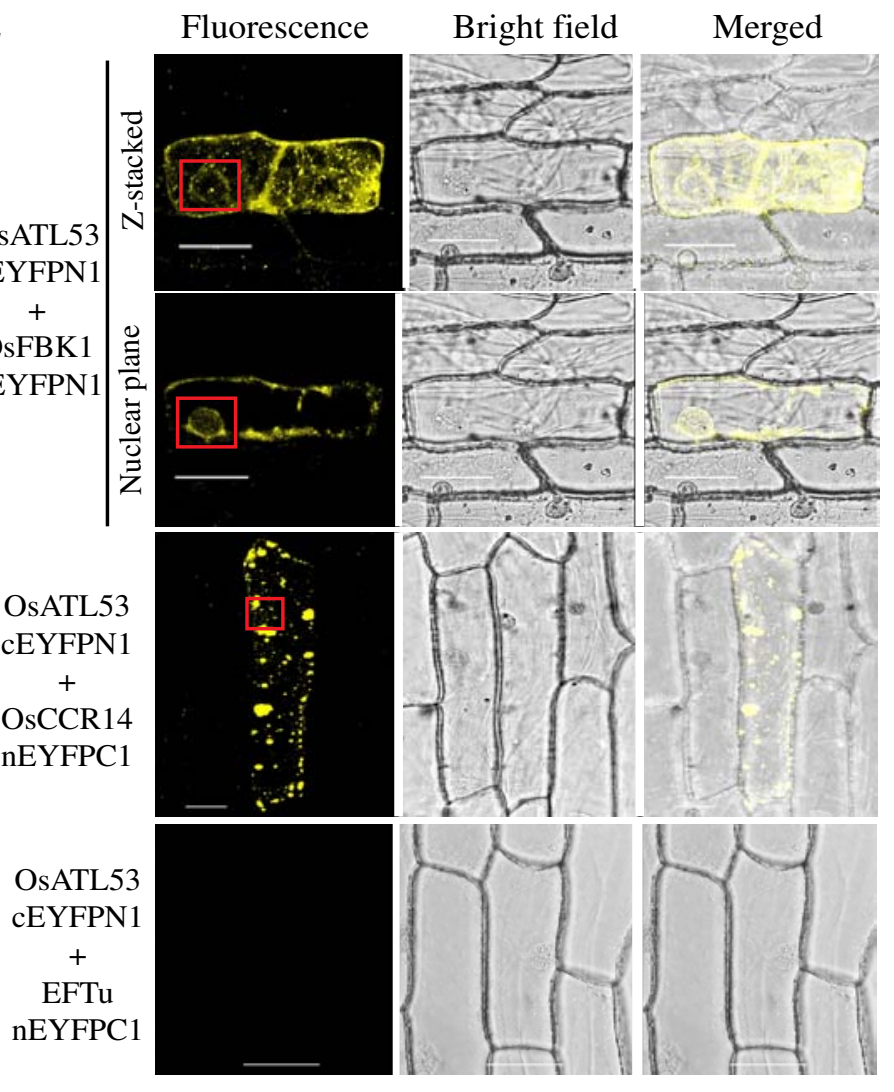

$50 \mathrm{kDa}-$

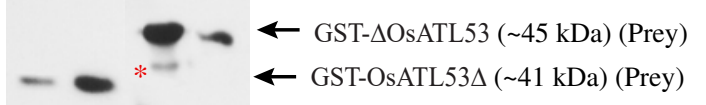

$\leftarrow \operatorname{GST}(\sim 26 \mathrm{kDa})$

$\leftarrow$ 6xHis-OsCCR14 ( $45 \mathrm{kDa})$ (Bait)
$\leftarrow$ 6xHis-OsFBK1 ( 47 kDa) (Bait)

Fig. 1. Interaction studies of OsATL53. A. In vitro immunoprecipitation assay between GST-CUL5g and 6xHis-OsATL53. Lanes 2, 3 and 4 are the unpurified input bacterial extracts of GST, GST-CUL5g and 6xHis-OsATL53, respectively. GST wash was used as a negative control for IP with 6xHis-OsFBK1 (lane 5). GST-tagged proteins were probed by rabbit anti-GST mAb, while 6xHis-OsATL53 was detected by using mouse anti-His mAb. These blots were parallelly processed with the same samples. M - Marker lane with the designated protein bands. B. In vitro auto-ubiquitintion assay to determine E3 property of OsATL53. Controls used are GST-OsATL53 $\Delta$ and GST- $\Delta$ OsATL53 respectively. Arrows determine the positions of ubiquitinated OsATL53/GST-OsATL53 $\Delta$ in top panel, red asterisks indicate non-specific bands. Bottom panel shows the input proteins as probed by anti-GST mAb. C. Representative image of cellular localization of OsATL53 in onion epidermal peel cells. Control: pSITE-3CA vector. Bright field images show the location of the nuclei in all panels. Bar- $50 \mu \mathrm{m}$. Red boxes denote nucleus. D. In vitro immunoprecipitation assay between GST-OsFBK1 and 6xHisOsATL53. GST wash (lane 2) was used as the negative control. Lanes 3 and 4 show the input bacterial extracts of GST-OsFBK1 and 6xHis-OsATL53 respectively. Lanes 5 and 6 are the IP lanes. E. YFP fluorescence showing the BiFC between OsATL53/OsFBK1 (upper panel) and OsATL53/OsCCR14 (middle panel) respectively in a representative cell. Bright field images show the location of the nuclei. Negative control: BiFC between OsATL53 and EFTu (lower panel). Bar- $50 \mu \mathrm{m}$. Red boxes denote nucleus. F. IP between GSTOsATL53 and 6xHis-OsCCR14. G. Western blot showing the IPs between GST- $\Delta$ OsATL53/ GST-OsATL53 $\Delta$ and OsCCR14 and OsFBK1 respectively. Red asterisks denote non-specifc binding. For all IPs, GST and GST-tagged proteins were probed by rabbit antiGST mAb, His-tagged proteins were detected by mouse anti-His mAb. M- marker lane for both blots. See also Fig. S1. 
OsCCR14 1CA $+$

OsATL53 3CA
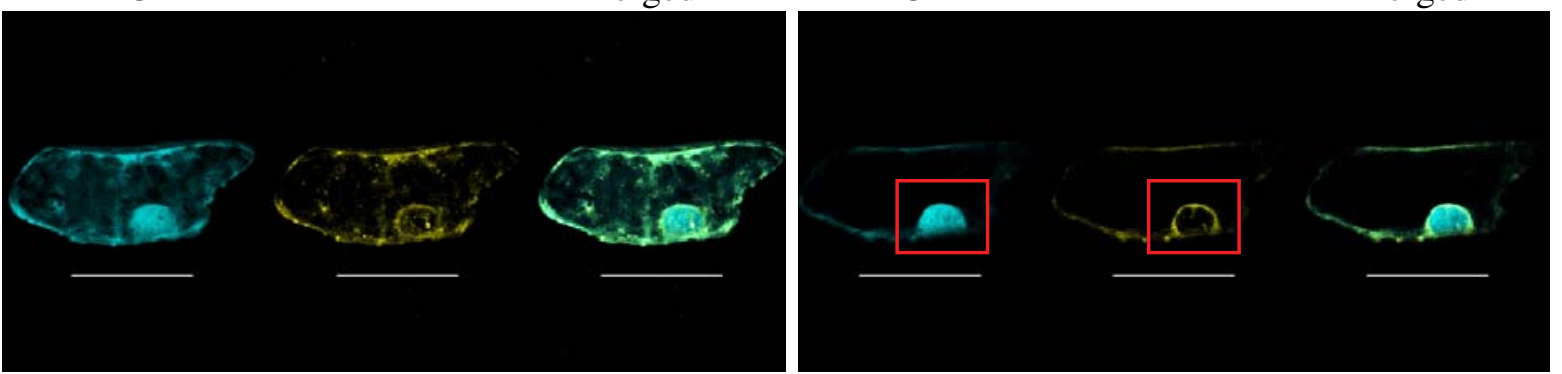

OsCCR14 1CA

$+$

OsATL53 3CA

$+$
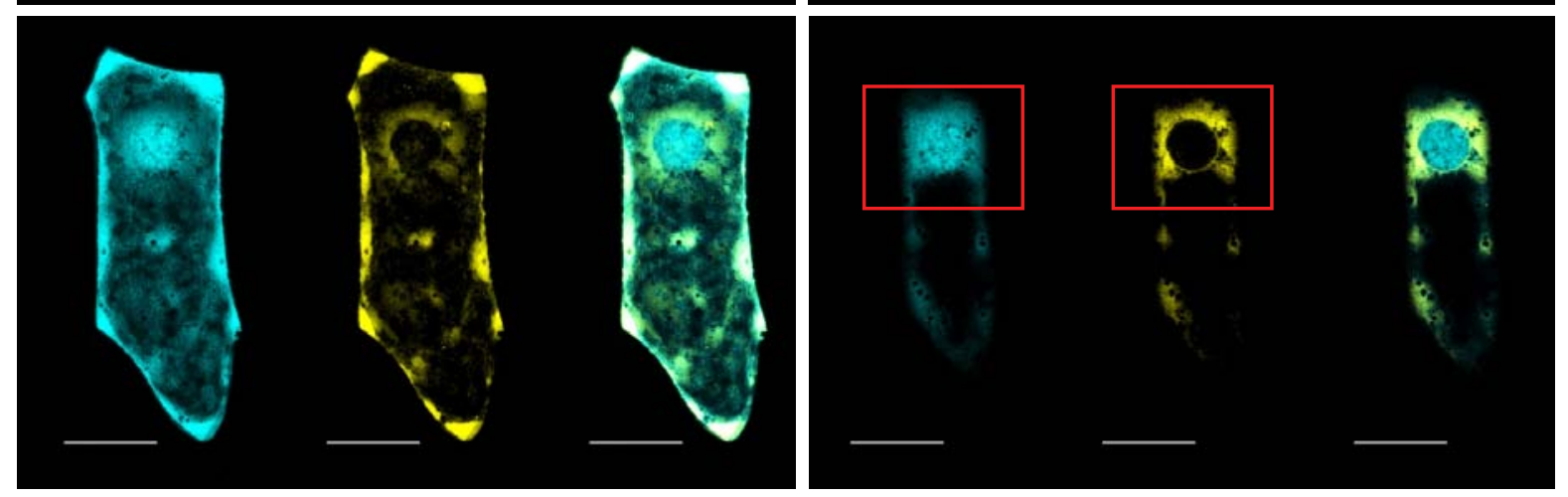

B

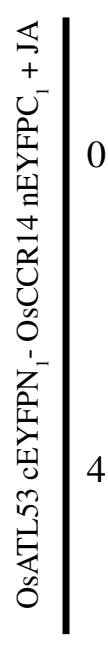

Fluorescence
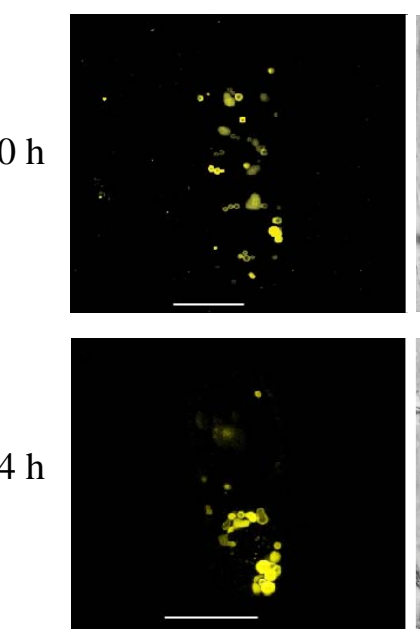
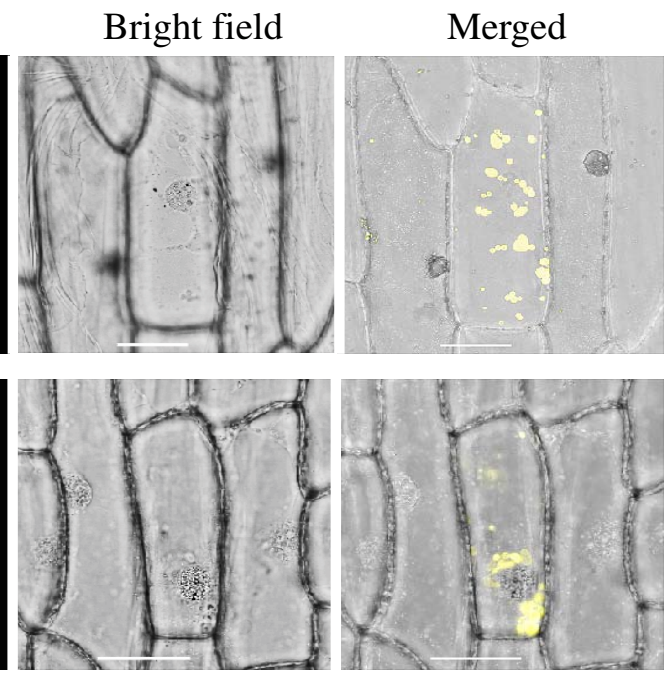

C

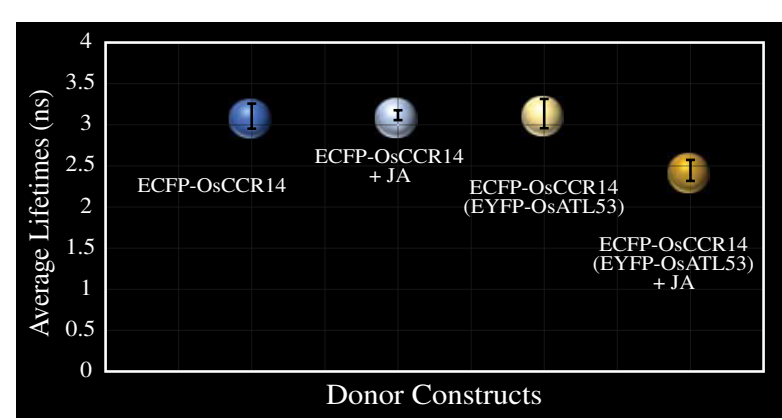

\begin{tabular}{|c|c|c|}
\hline $\begin{array}{c}\text { Donor } \\
\text { Constructs }\end{array}$ & $\begin{array}{c}\text { Average } \\
\text { Lifetimes } \\
\text { (ns) }\end{array}$ & $\mathrm{SD}$ \\
\hline $\begin{array}{c}\text { ECFP- } \\
\text { OsCCR14 }\end{array}$ & 3.1052 & 0.1523 \\
\hline $\begin{array}{c}\text { ECFP- } \\
\text { OsCCR14+ JA }\end{array}$ & 3.1165 & 0.0601 \\
\hline $\begin{array}{c}\text { ECFP-OsCCR14 } \\
\text { (EYFP-OsATL53) }\end{array}$ & 3.136 & 0.1780 \\
\hline $\begin{array}{c}\text { ECFP-OsCCR14 } \\
\text { (EYFP-OsATL53) } \\
\text { +JA }\end{array}$ & 2.4452 & 0.1277 \\
\hline
\end{tabular}
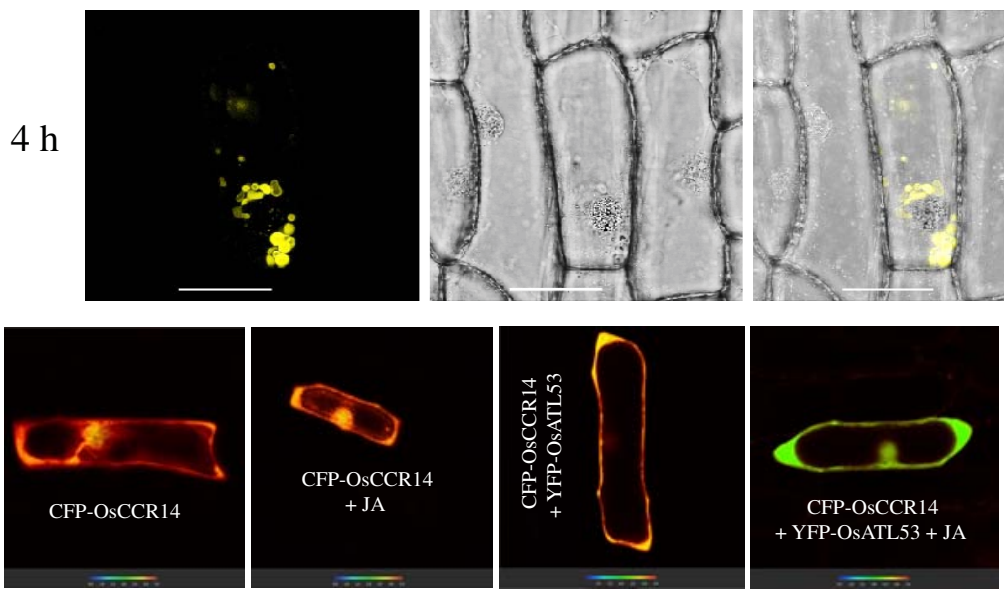

E

\section{Os}

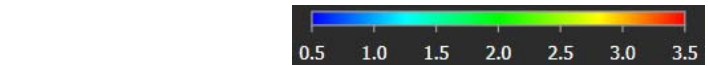

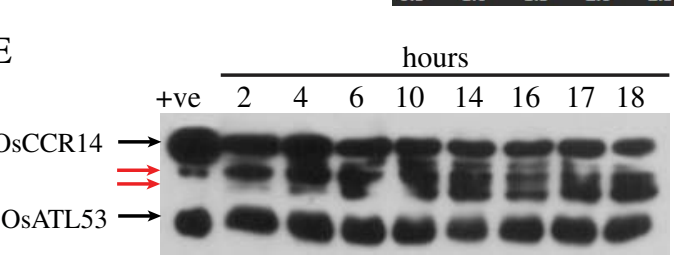

JA - MG132 WT cytoplasmic extract

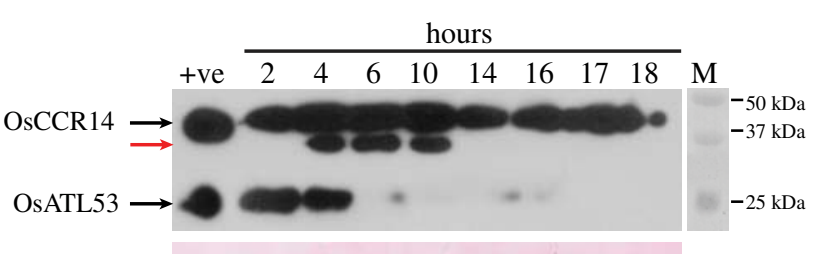

+ JA - MG132 WT cytoplasmic extract

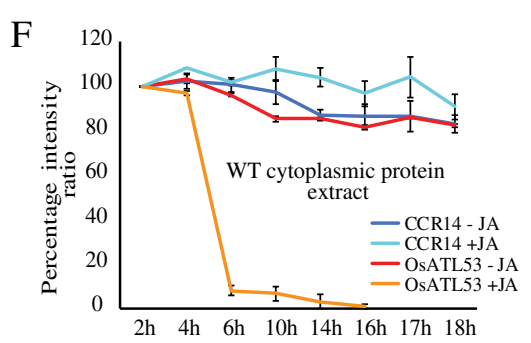

Fig. 3. Jasmonic acid induced changes in sub-cellular location and conformation of OsATL53-OsCCR14 complex. A. Co-localization of OsCCR14-ECFP and OsATL53-EYFP in onion peel cells. Upper panel shows ECFP and EYFP fluorescence without JA treatment, while lower panel shows changes in EYFP fluorescence after 4 hours of JA treatment. Red boxes show the accumulation of ECFP and EYFP fluorescence around the target cell's nucleus before and after JA treatment. B. Movement of OsATL53OsCCR14 BiFC complex in response to $6.4 \mathrm{mM}$ JA observed after 4 hours. Bright field images show the location of the nucleus in the representative target cell. Bar- $50 \mu \mathrm{m}$. C. Change in lifetime of ECFP-OsCCR14 donor construct upon exposure to JA under FLIM-FRET conditions. Error bars denote SD of at least 5 cells observed. D. Representative Fast FLIM images of target cells in normal and JA-induced conditions. Positive FRET is present through-out the cell. Bar represents the range of lifetimes from 0.5-3.5 ns. E. Cell-free degradation of 6xHis-OsCCR14 and 6xHis-OsATL53 in the cytoplasmic protein extracts of WT seedlings, in the absence and presence of JA respectively. Time kinetics performed in the absence of MG132. Loading control: Ponceau stained Rubisco large subunit band (55 kDa). Red arrows denote non-specific binding. M - marker lane. F. Graphical representation of cellfree degradation kinetics where error bars denote SD. See Fig. S3-S6. 
A
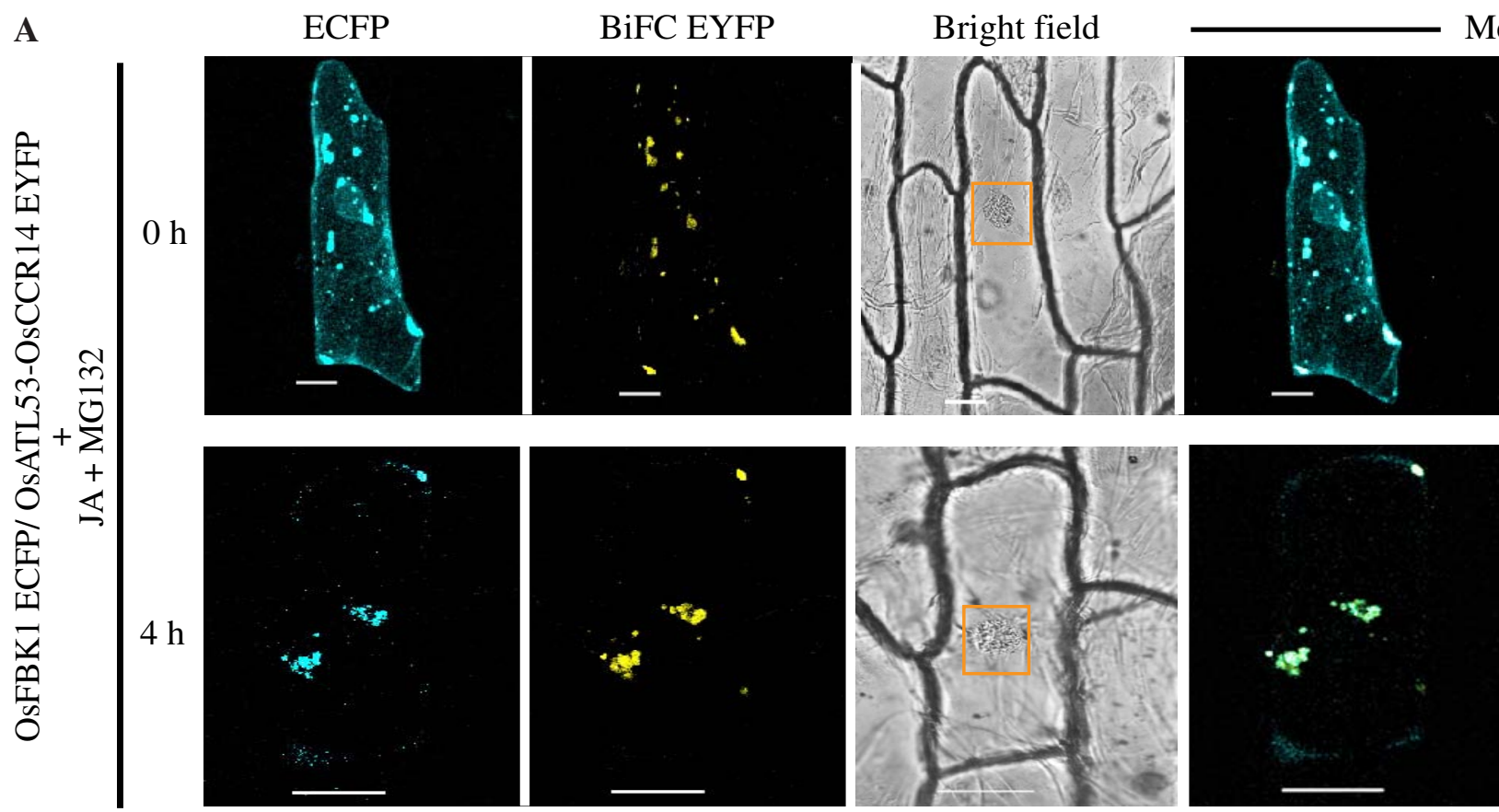

Merged
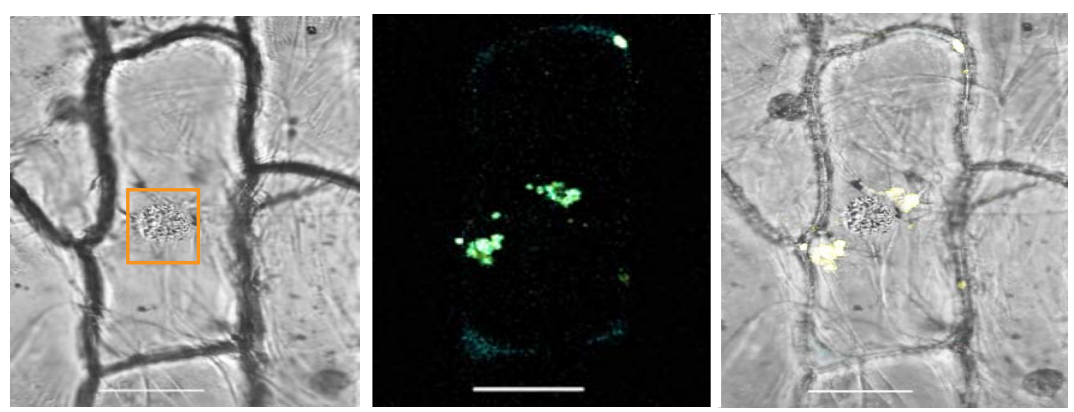

B
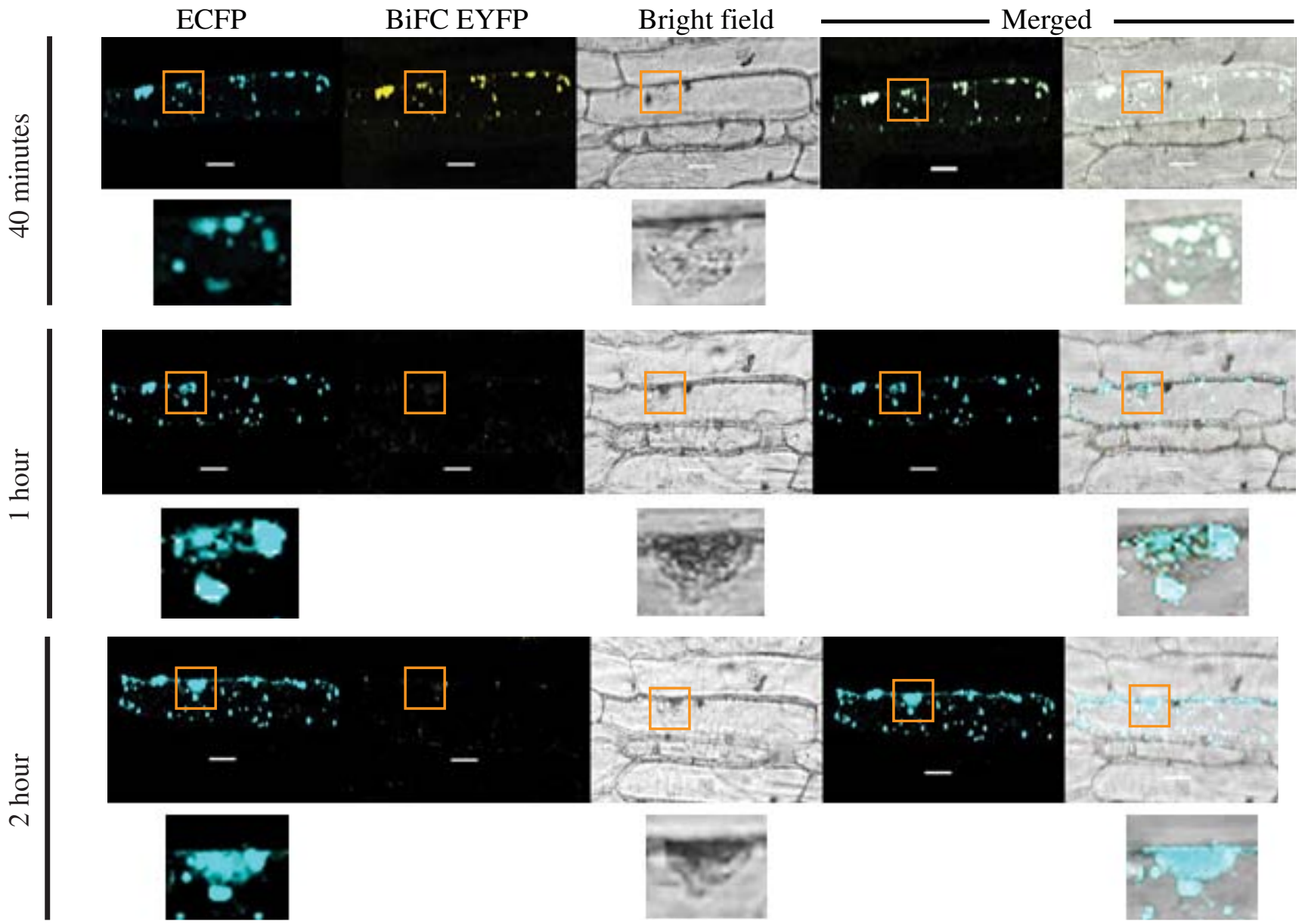

Fig. 4. Changes in protein interaction and localization dynamics in the presence of JA. A. OsFBK1 ECFP fluorescence was observed to merge with the BiFC (OsATL53-OsCCR14) EYFP fluorescence (Z-stacked) after 2 hours of JA exposure and accumulate around the nuclear boundary after 4 hours, in the presence of MG132. B. Changes in localization pattern of OsFBK1 after JA without MG132 treatment in presence of OsCCR14-OsATL53 complex. After degradation of OsATL53 and the disruption of the OsCCR14-OsATL53 complex and loss of EYFP signal, the normal localization pattern of OsFBK1 is seen. The same target cell was observed after the requisite time points after JA treatment. A loss in signal strength was observed due to repeated laser exposure, and the ECFP and EYFP fluorescence were adjusted accordingly. The orange boxes and inset pictures below each panel focusses the nucleus. Bright field images show the location of the nucleus in each target cell. Merged pictures are shown in both dark and bright field background. Green colour denotes overlapped regions of ECFP and EYFP fluorescence. Bar - $50 \mu \mathrm{m}$. See Fig. S7. 
A

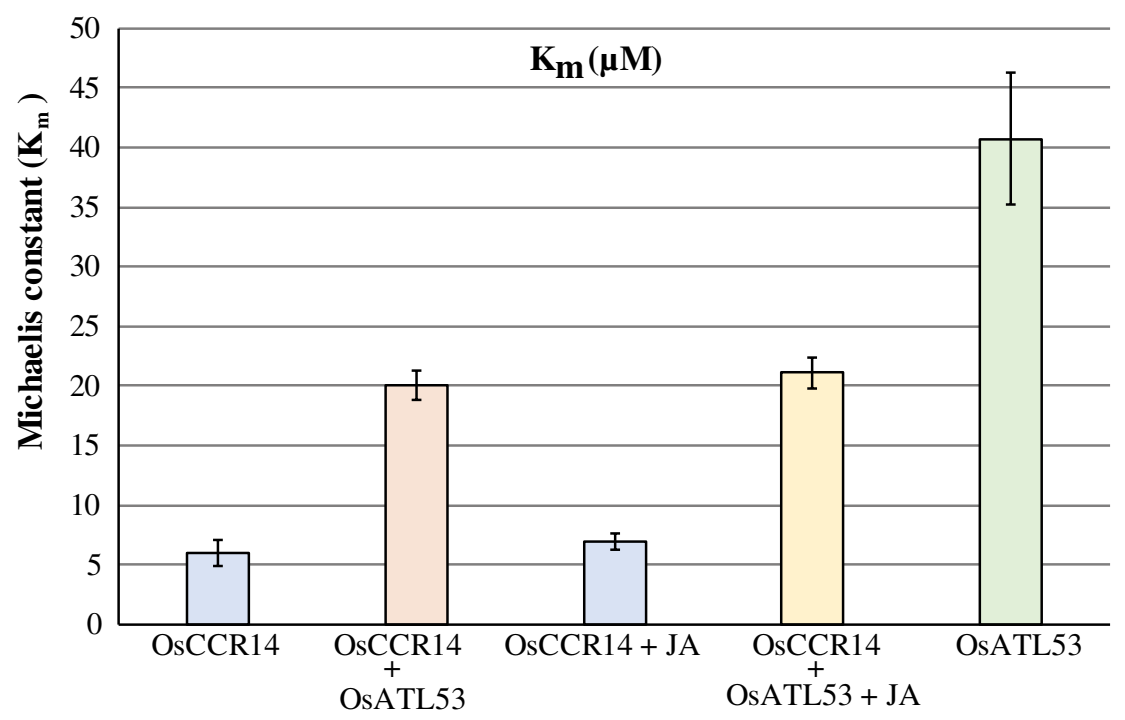

B

\begin{tabular}{|c|c|c|c|}
\hline Sample & $\mathbf{K}_{\mathbf{m}}(\boldsymbol{\mu M})$ & $\mathbf{K}_{\mathbf{c a t}}\left(\mathbf{s}^{-\mathbf{1}}\right)$ & $\mathbf{K}_{\mathbf{c a t}} / \mathbf{K}_{\mathbf{m}}\left(\mathbf{s}^{-\mathbf{1}} \boldsymbol{\mu M} \mathbf{1}^{\mathbf{1}}\right)$ \\
\hline OsCCR14 & $6.006 \pm 1.1075$ & $3.8978 \mathrm{e}-4 \pm 0.0029$ & $6.41 \mathrm{e}-5 \pm 4.45 \mathrm{e}-5$ \\
\hline OsCCR14 + OsATL53 & $20.0461 \pm 1.2642$ & $3.5456 \mathrm{e}-4 \pm 6.27 \mathrm{e}-5$ & $2.1234 \mathrm{e}-5 \pm 1.39 \mathrm{e}-5$ \\
\hline $\begin{array}{c}\text { OsCCR14 + JA } \\
\text { OsCCR14 + OsATL53 } \\
\text { + JA }\end{array}$ & $6.9571 \pm 0.7292$ & $2.28 \mathrm{e}-4 \pm 1.68 \mathrm{e}-5$ & $3.3124 \mathrm{e}-5 \pm 4.28 \mathrm{e}-6$ \\
\hline OsATL53 & $40.7378 \pm 5.5711$ & $2.8972 \mathrm{e}-4 \pm 1.79 \mathrm{e}-4$ & $1.4256 \mathrm{e}-5 \pm 9.12 \mathrm{e}-6$ \\
\hline
\end{tabular}

Fig. 5. Kinetic parameters of the enzymatic activities of OsCCR14 under different conditions. A: Graphical representation of the $\mathrm{K}_{\mathrm{m}}$ values of OsCCR14 either alone or in a complex with OsATL53, with or without JA. B: Tabular representation of the observed kinetic parameters of the various enzyme combinations. 

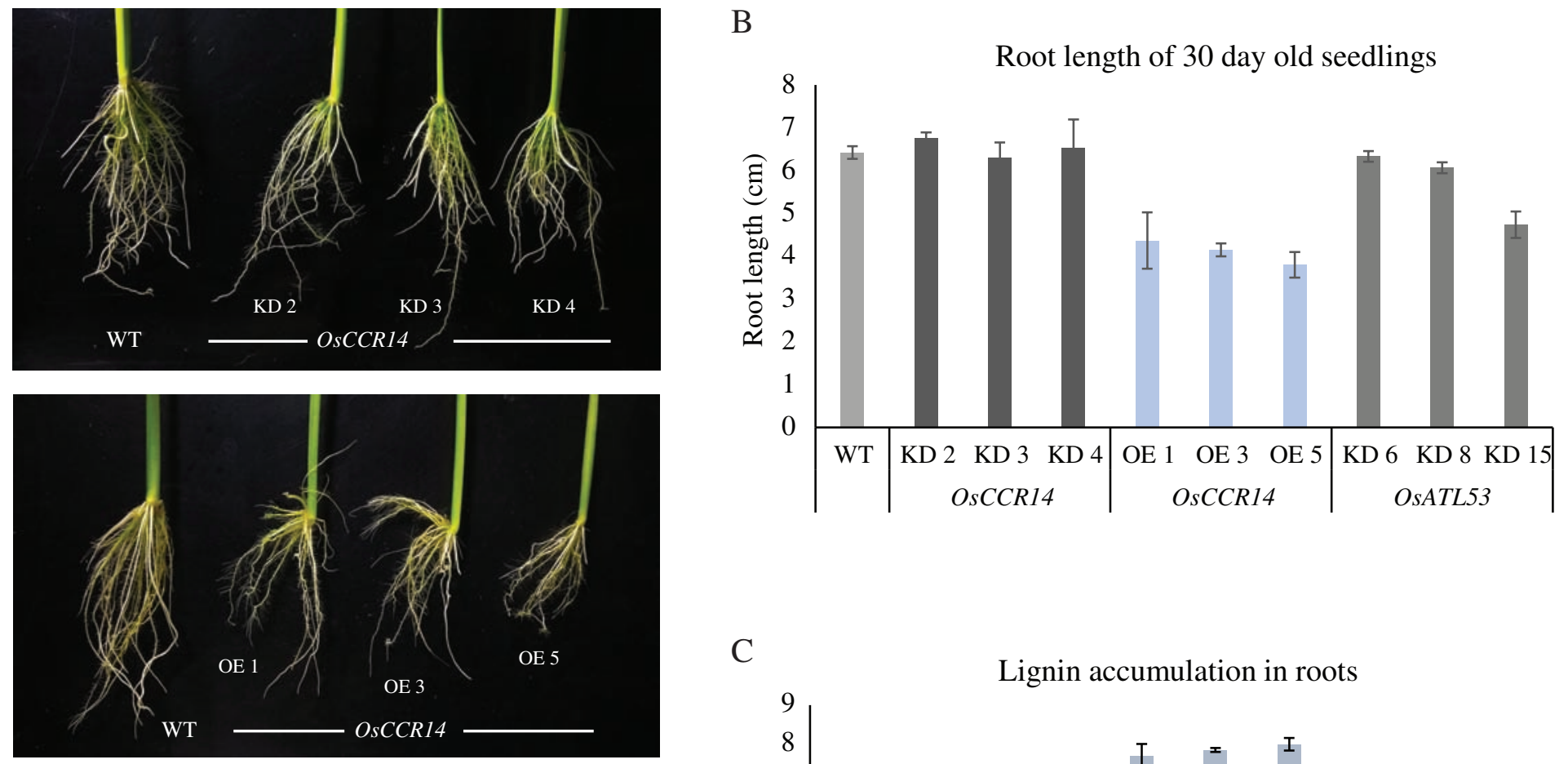

$\mathrm{C}$
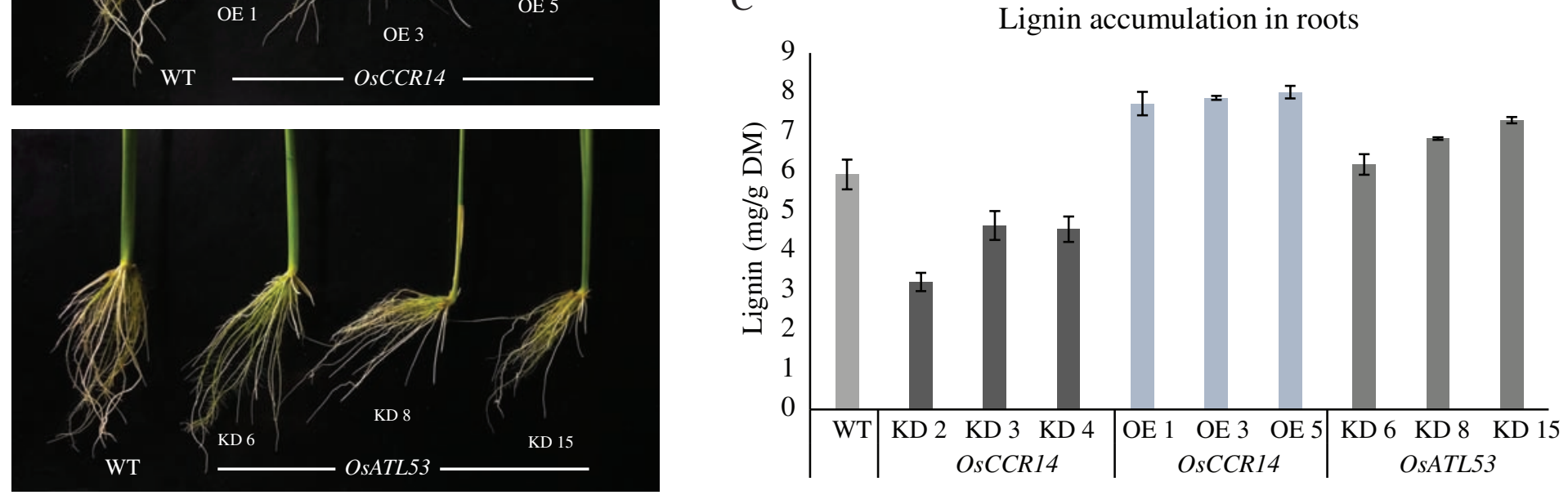

$\mathrm{D}$

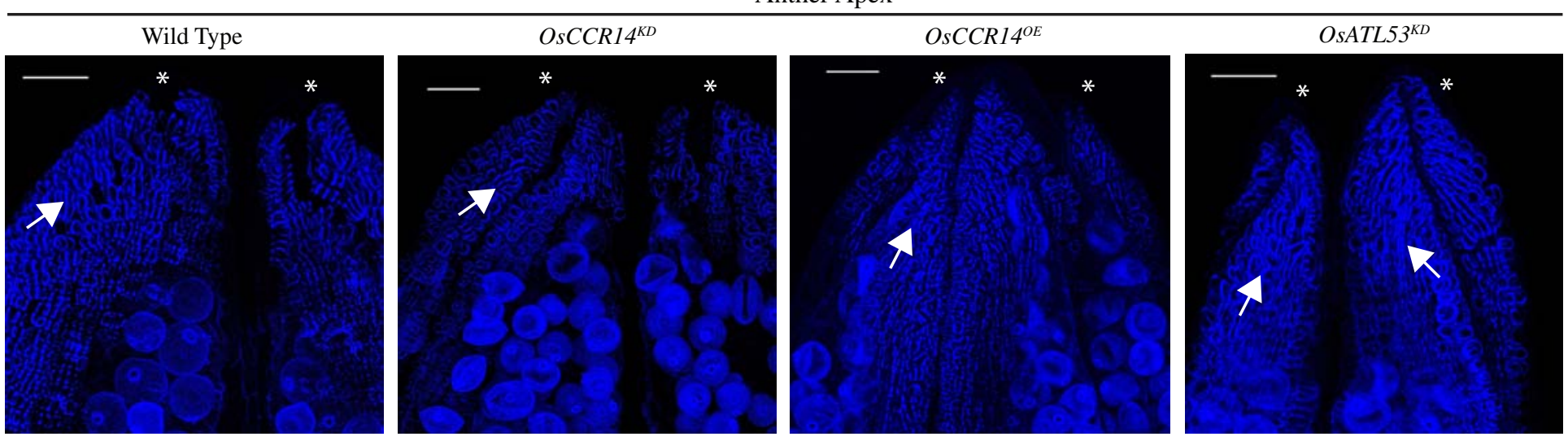

Anther Base
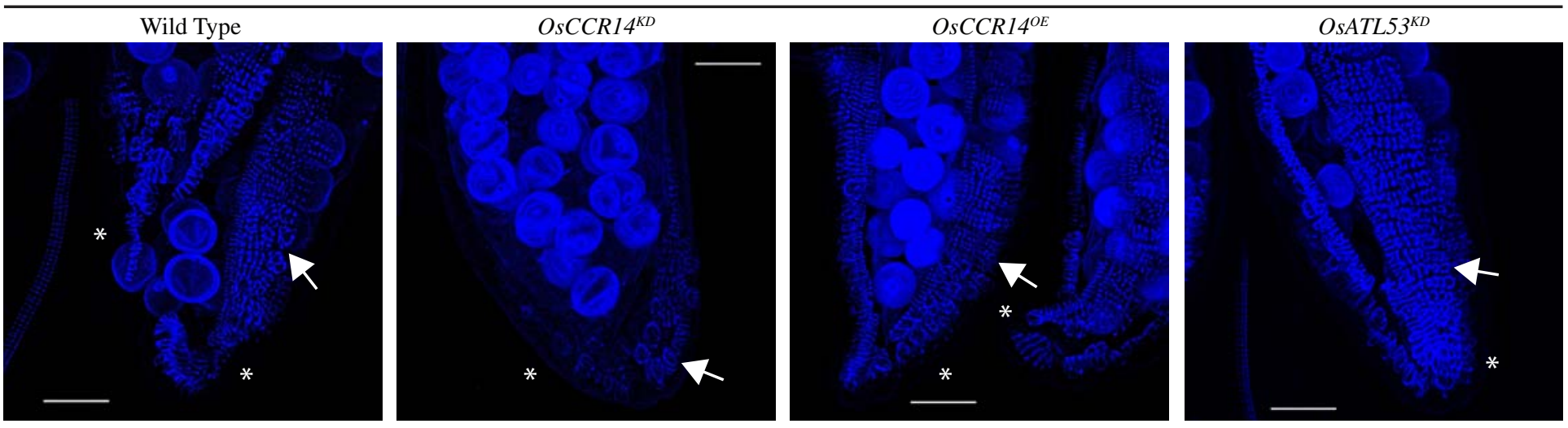

Fig. 6. Transgenic morphological data. A: Root morphology of 30-day-old seedlings. B: Graphical representation of root length of 30-day-old seedlings. Error bars - SD. C: Lignin estimation in roots of 30-day-old seedlings. Error bars - SD. D: Observation of wall morphology in representative rice pre-dehiscent anthers of wild type and transgenics. Confocal microscopy of cleared anther apices (top panels) and bases (bottom panels) of $O s C C R 14^{K D}$ and $O S A T L 53^{K D}$ transgenics and wild type showing the differences in the dispersal of the U-shaped wall thickenings in the endothecium. The U-shaped thickenings are indicated by arrows. Bar $=50 \mu \mathrm{m}$. Asterisk denotes separate locules of a single anther in the same frame. The globular structures are pollen grains. 


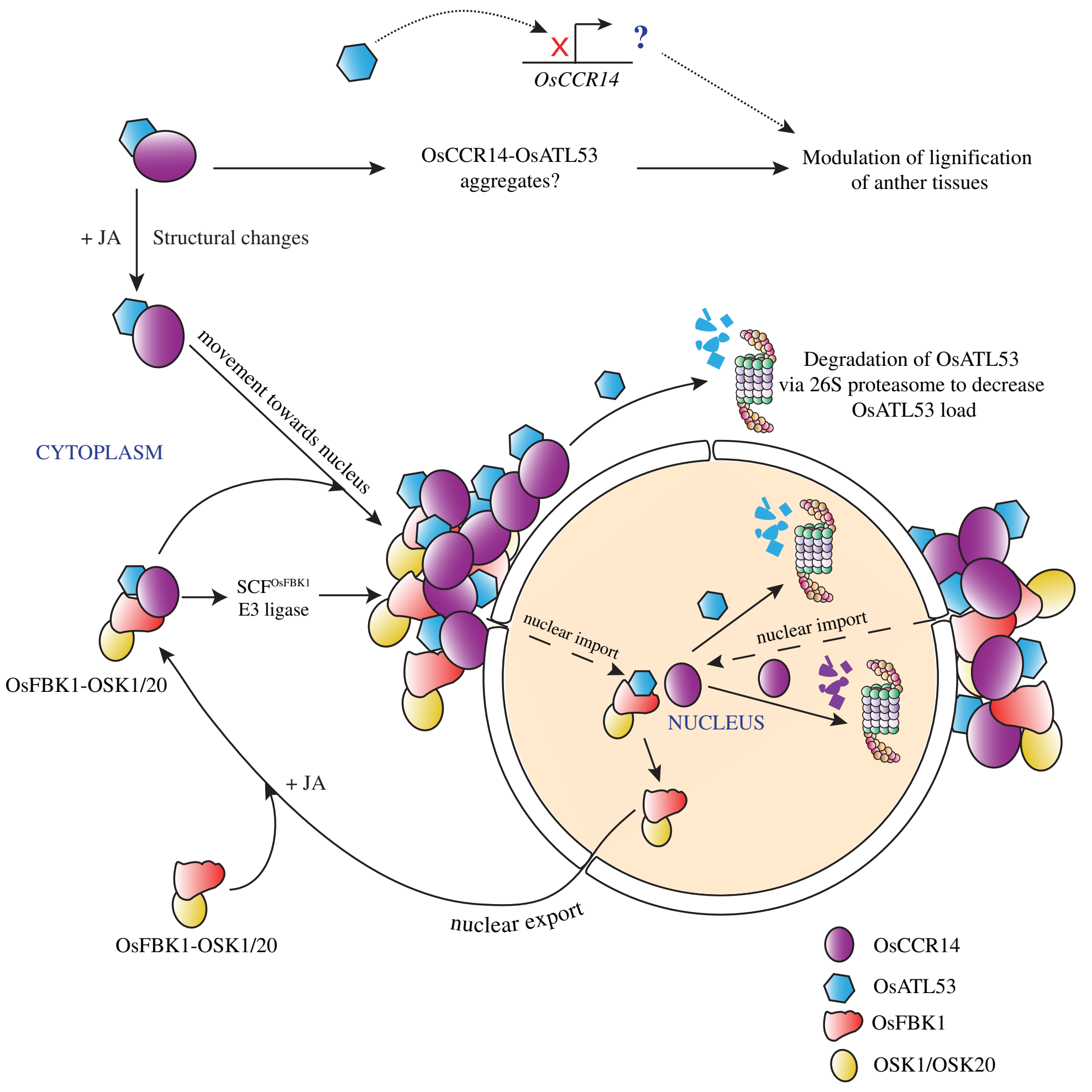

Fig. 7. Proposed model of the signalling pathway involved in the jasmonic acid-mediated degradation of OsATL53 and OsCCR14 by SCF ${ }^{\mathrm{OSFBK} 1}$ and the $26 \mathrm{~S}$ proteasome. OsFBK1 interacting with OSK1/OSK20 has been reported earlier (Borah and Khurana, 2018). See text for more details. 


\section{Supplementary Files}

This is a list of supplementary files associated with this preprint. Click to download.

- SupplementaryInformationNew.pdf 